\title{
Surface reconstruction of fluorites in vacuum and aqueous environment
}

\author{
Giuseppe Fisicaro, ${ }^{1, *}$ Michael Sicher, ${ }^{1}$ Maximilian Amsler, ${ }^{2}$ Santanu Saha, ${ }^{1}$ Luigi Genovese, ${ }^{3}$ and Stefan Goedecker ${ }^{1}$ \\ ${ }^{1}$ Department of Physics, University of Basel, Klingelbergstrasse 82, CH-4056 Basel, Switzerland \\ ${ }^{2}$ Laboratory of Atomic and Solid State Physics, Cornell University, Ithaca, New York 14850, USA \\ ${ }^{3}$ Laboratoire de simulation atomistique (L_Sim), SP2M, INAC, CEA-UJF, F-38054 Grenoble, France
}

(Received 23 June 2017; published 23 August 2017)

\begin{abstract}
Surfaces and interfaces of bulk materials with liquids are of importance for a wide range of chemical processes. In this work, we systematically explore reconstructions on the $(100)$ surface of calcium fluoride $\left(\mathrm{CaF}_{2}\right)$ and other fluorites $\left(M \mathrm{~F}_{2}\right), M=\{\mathrm{Sr}, \mathrm{Cd}, \mathrm{Ba}\}$ by sampling the configurational space with the minima hopping structure prediction method in conjunction with density functional theory calculations. We find a large variety of structures that are energetically very close to each other and are connected by very low barriers, resulting in a high mobility of the topmost surface anions. This high density of configurational states makes the $\mathrm{CaF}_{2}(100)$ surface a very dynamic system. The majority of the surface reconstructions found in $\mathrm{CaF}_{2}$ are also present in $\mathrm{SrF}_{2}, \mathrm{CdF}_{2}$, and $\mathrm{BaF}_{2}$. Furthermore, we investigate in detail the influence of these reconstructions on the crystal growth of $\mathrm{CaF}_{2}$ in solvents by modeling the fluorite-water interface and its wetting properties. We perform a global structural search both by explicitly including water molecules and by employing a recently developed soft-sphere solvation model to simulate an implicit aqueous environment. The implicit approach correctly reproduces both our findings with the explicit-water model and the experimentally reported contact angles for the partial-hydrophobic (111) and hydrophilic (100) surfaces. Our simulations show that the high anion mobility and the low coordination of the (100) surface atoms strongly favors the adsorption of water molecules over the (111) surface. The aqueous environment makes terminations with low-coordination surface atoms more stable, promoting (100) growth instead of the (111).
\end{abstract}

DOI: 10.1103/PhysRevMaterials.1.033609

\section{INTRODUCTION}

Fluorite-type crystals have been extensively investigated in the past [1-8] due to their broad technological relevance, including optical applications such as litho-excimer laser optics [9,10], beam deliveries, and zoom lenses, but also as a fluxing agent in the aluminum industry, as ceramic composites in fuel cell applications [11], and as an insulator within semiconductor multilayer structures [12]. Recently, $\mathrm{CaF}_{2}$ nanoparticles have also attracted interest for medical applications, e.g., as markers in cancer therapy [13] and for potential use in dental care [14-16]. As an ionic conductor, fluorites are promising candidates for solid state electrolytes in batteries: The prospect of a secondary battery based on a fluoride shuttle (fluoride ion battery, FIB) was discussed in Ref. [17]. Alkaline-earth $(\mathrm{Ca}, \mathrm{Ba}, \mathrm{Sr})$ fluorites together with several rare-earth counterparts have been proposed as electrolytes, both in their crystalline, nanocrystalline, doped, or nanostructured forms [1,18-20].

Understanding the structure of fluorite surfaces as well as their interfaces with wet environments is therefore of crossdisciplinary interest in physics, chemistry, and biology, in environmental, metallurgical, ceramic, and medical processes [21-23]. At an interface, many materials undergo reconstructions, i.e., an atomic rearrangement, leading to structural features that significantly differ from the crystalline bulk, strongly influencing their chemical properties like reactivity and solubility [24]. Hence, it is of utmost importance to investigate the underlying surface terminations of fluorites at the atomic level to fully characterize their properties. However,

\footnotetext{
*giuseppe.fisicaro@unibas.ch
}

resolving the atomic structure of surface reconstructions experimentally is challenging, especially for highly reactive surfaces [5] or in the presence of a wet environment [24-29]. $A b$ initio simulations are particularly valuable in this context, allowing an accurate modeling of these systems as a function of varying external conditions. In fact, density functional theory (DFT) has meanwhile become the main workhorse for atomistic simulations, providing a high predictive power for structural properties in material science at a convenient computational cost.

Fluorite growth strongly depends on the surrounding environment. Growth in vacuum occurs towards $\{111\}$ facets [5]. On the other hand, naturally grown fluorite in wet environments predominantly adopts a cubic $\{100\}$ shape [30-32]. Similarly, the synthesis of $\mathrm{CaF}_{2}$ micro- and nanoparticles $[33,34]$ under certain hydrothermal conditions of $\mathrm{pH}$, temperature and concentration of precursor solutions [16] also leads to cubic morphologies. This behavior is surprising since the Gibbs-Wulff theorem [35] appears to be violated, which states that nanocrystals are to adopt the shape which results in the smallest total surface energy, i.e., $\min \left(\sum_{i} \gamma_{i} A_{i}\right)$, where $\gamma_{i}$ denotes the surface energy of the $i$ th face and $A_{i}$ denotes its surface area. Since the most stable surface of $\mathrm{CaF}_{2}$ is the (111) face [36,37], which is the natural cleavage plane, nanoparticles are expected to adopt an octahedral rather than a cubic shape.

A hypothetical cleaving along the $\{100\}$ planes would lead to a stacking sequence of alternately charged planes [38] resulting in a dipole moment perpendicular to the surface and to very high surface energies in crystalline samples [39]. The resulting dipole moment can be eliminated by removing every second F ion on an F-terminated (100) surface or every second $\mathrm{Ca}$ ion on a Ca-terminated surface. Nevertheless, the surface energy remains higher than the (111) face and a 
substantial reconstruction is expected [40], which has however, to our knowledge, never been systematically investigated. The adsorption of a mono layer or thin film of water as well as other adsorbates could potentially lead to a stabilization of the surface [41], a mechanism that has also not been studied before. During such an adsorption/desorption process, the surface could undergo further reconstructions, leading to an additional reduction of its energy in an aqueous environment.

In this paper, we present a comprehensive study of the surface reconstructions in ionic materials through an unbiased systematic structural search together with first-principle electronic-structure calculations at the DFT level. Starting from $\mathrm{CaF}_{2}$, we extend our investigation to other ionic systems that crystallize in the fluorite structure with $F m \overline{3} m$ symmetry, i.e., $\mathrm{SrF}_{2}, \mathrm{CdF}_{2}$, and $\mathrm{BaF}_{2}$.

Besides exploring the surface reconstruction in vacuum, we also study the adsorption mechanism of water on the (100) and (111) surfaces of $\mathrm{CaF}_{2}$ by explicitly including water molecules in the simulation domain. Different degrees of surface coverage are explored through a global structural search for the solid-liquid interface. This explicit inclusion of water should be in principle the most accurate to account for solvent effects, albeit fully explicit $a b$-initio approaches result in well-known limitations in describing an aqueous environment, in particular regarding its structural and dielectric properties [42-44]. In order to reduce the large number of degrees of freedom of the explicit solvent model, which gives many different configurations of the water molecules for identical surface reconstructions, we employ in addition a recently developed implicit solvation model $[45,46]$. Using this method, we explore the potential energy surface of the hydrated surface reconstructions. The wetting properties in terms of the water contact angle and spreading coefficient are investigated as well and compared to experimental results.

The manuscript is structured as follows: Section II presents the methods and tools employed in this work, reporting the analysis of the cleaved (111) and (100) $\mathrm{CaF}_{2}$ surfaces. In Sec. III, the various structures of the (reconstructed) surfaces and their stability are described in detail. Their dynamical properties and the energy barriers connecting the different configurations are also explored. The surface stability in the presence of an aqueous environment, the adhesion, and wetting properties are discussed in Sec. IV. The final results are summarized in Sec. V.

\section{METHOD AND CLEAVED SLABS}

The minima hopping method (MHM) [47,48] was employed to sample low energy surface reconstructions by systematically exploring the potential energy surface (PES). Within the MHM, consecutive short molecular dynamics (MD) simulations are performed to escape from local minima, followed by local geometry optimizations to efficiently sample the energy landscape. The initial velocities of the MD escape trials are preferably aligned along low curvature modes of the local minima to exploit the Bell-Evans-Polanyi principle [49]. A feedback mechanism based on a search history discourages revisiting already known local minima, allowing a fast exploration of large portions of the PES. Recently, the MHM was successfully applied in similar applications, for example to
TABLE I. Lattice constant $a_{0}$ and bulk modulus $B_{0}$ for $\mathrm{CaF}_{2}$, $\mathrm{SrF}_{2}, \mathrm{CdF}_{2}$, and $\mathrm{BaF}_{2}$ fluorites. Both our DFT calculations with the $\mathrm{PBE}$ functional as well as the experimental data are reported.

\begin{tabular}{lccccc}
\hline \hline & \multicolumn{2}{c}{$a_{0}[\AA ⿻$} & & \multicolumn{2}{c}{$B_{0}[\mathrm{GPa}]$} \\
\cline { 2 - 3 } \cline { 5 - 6 } fluorides & DFT/PBE & Expt. & & DFT/PBE & Expt. \\
\hline $\mathrm{CaF}_{2}$ & 5.50 & $5.46^{\mathrm{a}}$ & & 77.92 & $82 \pm 0.7^{\mathrm{b}}, 87 \pm 5^{\mathrm{a}}$ \\
$\mathrm{SrF}_{2}$ & 5.85 & $5.80^{\mathrm{c}}$ & & 64.55 & $69.7 \pm 1.1^{\mathrm{d}}$ \\
$\mathrm{CdF}_{2}$ & 5.48 & $5.39^{\mathrm{e}}$ & & 89.61 & \\
$\mathrm{BaF}_{2}$ & 6.23 & $6.20^{\mathrm{c}}$ & & 55.06 & $56.9 \pm 0.9^{\mathrm{d}}$ \\
\hline \hline
\end{tabular}

${ }^{\mathrm{a}}$ Reference [63]; ${ }^{\mathrm{b}}$ Reference [64]; ${ }^{\mathrm{c}}$ Reference [65]; ${ }^{\mathrm{d}}$ Reference [66]; e Reference [67].

predict reconstructions of boron surfaces [50] and to investigate the surfaces of atomic force microscopy silicon model tips $[51,52]$. The transition states between the local minima on the PES were explored using the bar-saddle method [53], which implements a double ended saddle point search algorithm.

All structural searches were performed directly at the DFT level by coupling the MHM with the BigDFT package [54-56]. BigDFT uses wavelets as basis functions, which are localized both in real and Fourier space and allow for an exact treatment of the surface boundary conditions without the need to introduce vacuum regions in the nonperiodic dimensions. Soft norm-conserving Hartwigsen-Goedecker-Hutter pseudopotentials $[57,58]$ were used to treat the core electrons within the local density approximation (LDA) and the generalized gradient approximation (PBE) [59] to the exchangecorrelation potential as implemented in the Libxc [60] library.

Careful tests were carried out to ensure the convergence of the DFT results. The wavelet basis functions were distributed on an adaptive uniform mesh with a resolution of $h_{\text {grid }}:=$ $h_{x}=h_{y}=h_{z}=0.25$ Bohr for all calculations. A $4 \times 4 \times 4$ Monkhorst-Pack $k$-point mesh was used for the bulk material in the conventional cell, resulting in energies converged to within $1 \mathrm{meV}$ per atom. To assess the accuracy of the pseudopotentials, the lattice constants and bulk moduli of the pristine crystalline cells of $\mathrm{CaF}_{2}, \mathrm{SrF}_{2}, \mathrm{CdF}_{2}$, and $\mathrm{BaF}_{2}$ were computed and compared to the experimental values $[61,62]$, as summarized in Table I. For $\mathrm{CaF}_{2}$ with LDA we obtained a value of $a_{0, \mathrm{LDA}}=$ $5.33 \AA$ for the lattice constant and a bulk modulus $B_{0}$ of 102.25 $\mathrm{GPa}$. As expected, LDA underestimates the lattice constant of $\mathrm{CaF}_{2}$ by about $2.4 \%$, whereas PBE leads to a slight overestimation by $0.73 \%$. Similarly, the LDA bulk modulus is higher than the experimental value, while the $\mathrm{PBE}$ results are much closer to experiments. For $\mathrm{SrF}_{2}, \mathrm{CdF}_{2}$, and $\mathrm{BaF}_{2}, \mathrm{PBE}$ overestimates the lattice constants slightly by $0.85 \%, 1.64 \%$, and $0.48 \%$, respectively. PBE generally underestimates the bulk moduli, however, by less than $10 \%$. Overall, the PBE functional performs very well, as expected for simple ionic materials.

\section{A. (111) $\mathrm{CaF}_{2}$ surface}

In our study, we used a supercell to model the (111) surface of $\mathrm{CaF}_{2}$, as shown in Fig. 1. The fluorine anions are represented by small red (dark) spheres and the calcium cations by large gray (light) spheres, a convention that applies to all figures in the present work. The supercell is constructed from the cell 


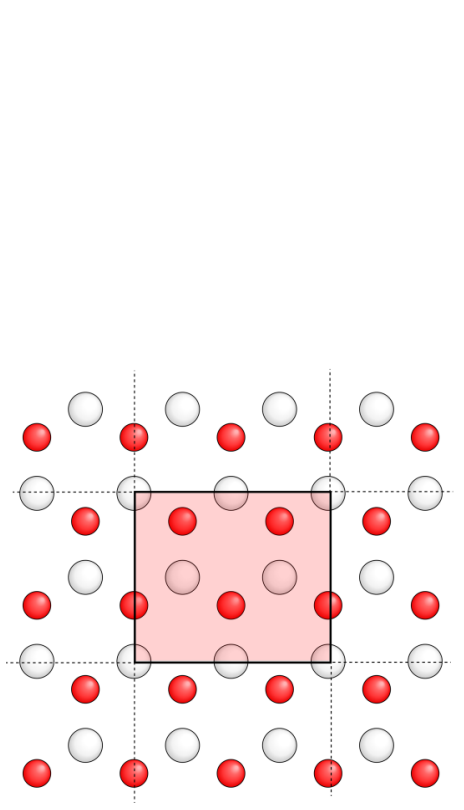

(a)

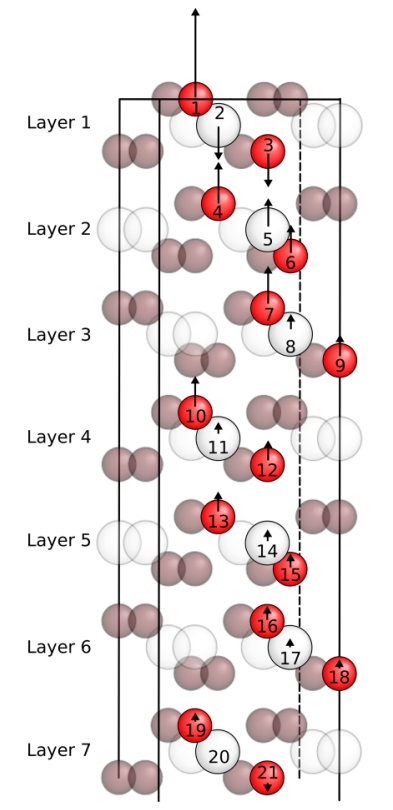

(b)

FIG. 1. (a) Top view onto the (111) $\mathrm{CaF}_{2}$ surface (topmost two layers). The outermost fluorine anion layer is denoted with small dark (red) spheres, and the calcium cation layer just below is denoted with large light (gray) spheres. The cross sectional area of the orthogonal supercell is shaded (red). (b) The top seven (triple-) layers of the relaxed (111) supercell consisting of 13 (triple-) layers and 156 atoms. Numbered and highlighted atoms are related to the analysis of the atomic displacement $\Delta z$ shown in Fig. 2. Arrows indicate the direction of the atomic displacements after relaxation, and their length qualitatively represents the displacement amplitude with respect to their bulk positions.

vectors $\{\mathbf{a}, \mathbf{b}, \mathbf{c}\}$ of the conventional unit cell as follows:

$$
\begin{aligned}
& \mathbf{a}^{*}=\frac{\sqrt{2}}{6}[(\sqrt{3}+3) \cdot \mathbf{a}+(\sqrt{3}-3) \cdot \mathbf{b}-2 \sqrt{3} \cdot \mathbf{c}] \\
& \mathbf{b}^{*}=\frac{\sqrt{3}}{6 \sqrt{2}}[(\sqrt{3}-3) \cdot \mathbf{a}+(\sqrt{3}+3) \cdot \mathbf{b}-2 \sqrt{3} \cdot \mathbf{c}] \\
& \mathbf{c}^{*}=\alpha(\mathbf{a}+\mathbf{b}+\mathbf{c}),
\end{aligned}
$$

where $\alpha$ is the thickness of the slab in the nonperiodic direction. The area of the slab cross section is given by $\sqrt{3} \cdot a_{0}^{2}$, where $a_{0}$ is the bulk lattice constant.

A convergence test with respect to the $k$-point sampling of the slab geometry shows that a $2 \times 2 \times 1 k$-point mesh and a uniform wavelet grid with $h_{\text {grid }}=0.25$ Bohr is sufficient for our supercell, resulting in a surface energy with an accuracy within $1 \mathrm{~mJ} / \mathrm{m}^{2}$. This parameter set was therefore used throughout the rest of our work to provide comparable results through error cancellation. The atomic positions were relaxed until all forces were smaller than $5 \mathrm{meV} / \AA$. Thanks to our Poisson solver, which makes use of interpolating scaling functions, and a wavelet-based treatment of the KohnSham Hamiltonian, BigDFT allows for an exact treatment of surface boundary conditions. As a consequence, spurious electrostatic interactions between slabs do not arise in our surface calculations, making our method ideally suited to

TABLE II. Surface energies $\left[\mathrm{J} / \mathrm{m}^{2}\right]$ of the relaxed (111) and (100) $\mathrm{CaF}_{2}$ surfaces for different slab thickness, calculated using Eq. (1).

\begin{tabular}{lcc}
\hline \hline & LDA & PBE \\
\hline (111) surface & & \\
5 triple-layer $\gamma_{(111)}$ & 0.558 & 0.366 \\
9 triple-layer $\gamma_{(111)}$ & 0.559 & 0.365 \\
13 triple-layer $\gamma_{(11)}$ & 0.560 & 0.365 \\
(100) surface with F termination & & \\
7 layer $\gamma_{(100)}$ & 1.205 & 0.856 \\
11 layer $\gamma_{(100)}$ & 1.160 & 0.795 \\
15 layer $\gamma_{(100)}$ & 1.153 & 0.781 \\
19 layer $\gamma_{(100)}$ & 1.152 & 0.778 \\
(100) surface with Ca termination & & \\
13 layer $\gamma_{(100)}$ & 1.539 & 1.211 \\
\hline \hline
\end{tabular}

address electronic-structure calculations of polarized systems with high efficiency and accuracy.

The surface energy $\gamma_{(h k l)}$ is calculated according to

$$
\gamma_{(h k l)}=\frac{1}{2 A}\left(E_{\text {slab }}^{\text {relaxed }}-n E_{\text {bulk }}\right)
$$

where $A=\left|\mathbf{a}^{*}\right|\left|\mathbf{b}^{*}\right|$ is the slab's cross section area and $E_{\text {slab }}^{\text {relaxed }}$ is the total energy of the relaxed slab. $n$ is the number of formula units within the slab and $E_{\text {bulk }}$ is the energy of the bulk system per formula unit.

Converged values of the total slab energy $E_{\text {slab }}^{\text {relaxed }}$ were obtained for a fully relaxed slab consisting of 13 (triple-) layers (see Table II) both with the LDA and PBE functionals, resulting in surface energies of 0.560 and $0.365 \mathrm{~J} / \mathrm{m}^{2}$, respectively. In comparison, Puchin et al. [68] reported values of $\gamma_{(111)}=$ $0.47 \mathrm{~J} / \mathrm{m}^{2}$ using the Hartree-Fock method with Gaussian basis sets. H. Shi et al. [37] reported $\gamma_{(111)}=0.438 \mathrm{~J} / \mathrm{m}^{2}$ using the hybrid B3PW exchange-correlation functional. Concerning the structure, we only found very small displacements $\Delta z$ of the atoms in the (111) surface after relaxation, i.e., $|\Delta z| / a_{0, \mathrm{PBE}}<0.3 \%$ (where $|\Delta z|=\left|z-z_{\text {bulk }}\right|$ ) as shown in Fig. 2, in strong contrast to the reports of H. Shi et al. All ions within the same sublayer relax by the same amount and into the same direction. Only the outmost F ions relax significantly by $0.014 \AA$. Very similar values were obtained with the LDA functional and are consistent with results of Puchin et al. [68], where the displacement $\Delta z$ is almost negligible and less than $0.01 \AA$ for all (sub-) layers. The weak relaxation leads to a small difference in energy of the unrelaxed and the relaxed slab of merely $0.05 \%$.

\section{B. (100) $\mathrm{CaF}_{2}$ surface}

For the (100) surface, we considered a supercell with a surface area twice as large as the area of the conventional cell. The cell vectors $\left\{\mathbf{a}^{*}, \mathbf{b}^{*}, \mathbf{c}^{*}\right\}$ are given by

$$
\begin{aligned}
& \mathbf{a}^{*}=\mathbf{a}+\mathbf{b} \\
& \mathbf{b}^{*}=-\mathbf{a}+\mathbf{b} \\
& \mathbf{c}^{*}=\alpha \mathbf{c}
\end{aligned}
$$

with $\alpha$ giving the slab thickness. An F-terminated slab representing the (100) surface is constructed from the bulk 


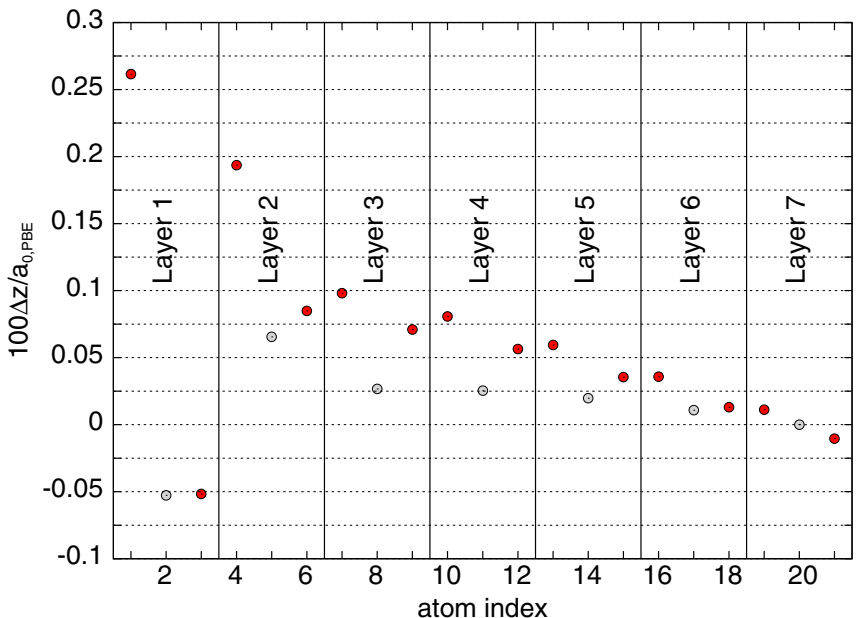

FIG. 2. Inward/outward relaxation (PBE) amplitudes with respect to the bulk positions in units of $\% a_{0, \mathrm{PBE}}$ for the topmost layers of the (111) $\mathrm{CaF}_{2}$ supercell. Negative values indicate an inward displacement (towards the bulk); positive values indicate a displacement towards the surface. Atom indexes are related to Fig. 1(b).

material by removing every second $\mathrm{F}$ anion from the outermost layers to achieve charge neutrality and to eliminate the dipole moment of this stacking sequence (see Fig. 3). This finally leads to a first reconstruction and a $c(2 \times 2) \mathrm{F}$ layer.

A Ca-terminated $c(2 \times 2)$ slab is constructed following the procedure by Hartman [40]: Every second top/bottom layer cation is removed, leading to a square lattice cation arrangement of the top/bottom layers (see Fig. 4)

Similar to the $(111)$ surface, a $(2 \times 2 \times 1) k$-point mesh was found to give sufficiently converged total energies for the (100) slab. Table II summarizes the surface energies for both terminations at varying slab thickness. Slabs consisting of 15 and 19 single layers for the $\mathrm{F}$ termination and 13 single layers for $\mathrm{Ca}$ termination result in converged surface energies. We found surface energies of 1.152 and $0.778 \mathrm{~J} / \mathrm{m}^{2}$ for the F-terminated slab with LDA and PBE, respectively. Reference [37] reported a value of $\gamma_{(100)}=0.957 \mathrm{~J} / \mathrm{m}^{2}$, while Ref. [68] found $\gamma_{(100)}=1.189 \mathrm{~J} / \mathrm{m}^{2}$, the latter agreeing well with our findings using LDA. The atomic displacements $\Delta z$ after relaxation of the F-terminated (100) slab is shown in Fig. 5(a). The displacements are considerably larger than for the (111) surface. In particular, the displacement of the toplayer $\mathrm{F}$ ions is $3.9 \% a_{0, \mathrm{PBE}}$ inwards. In contrast, the $\mathrm{F}$ ions in layers 3 and 5 move into the opposite directions: The outward displacement by $4.2 \% a_{0, \mathrm{PBE}}$ in layer 3 is even larger than the inward movement in the top layers. The cations in the second layer move by $1.2 \% a_{0, \mathrm{PBE}}$ outwards. The atomic displacements of the Ca-terminated slab is shown in Fig. 5(b). A significant atomic displacement of the Ca-terminated slab only occurs for the top layer cations, which move by $8.5 \%$ $a_{0, \mathrm{PBE}}$ inwards while all other layers move by merely less than $1 \% a_{0, \mathrm{PBE}}$. The computed surface energy of the Ca-terminated slab is 1.539 and $1.211 \mathrm{~J} / \mathrm{m}^{2}$ with LDA and PBE, respectively. In both cases, an F termination of the (100) surface is clearly favored over a termination with $\mathrm{Ca}$ atoms.

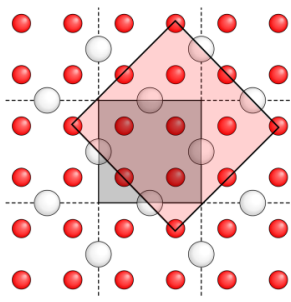

(a)

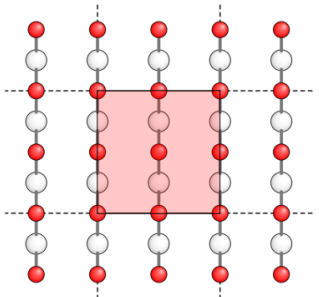

(b)

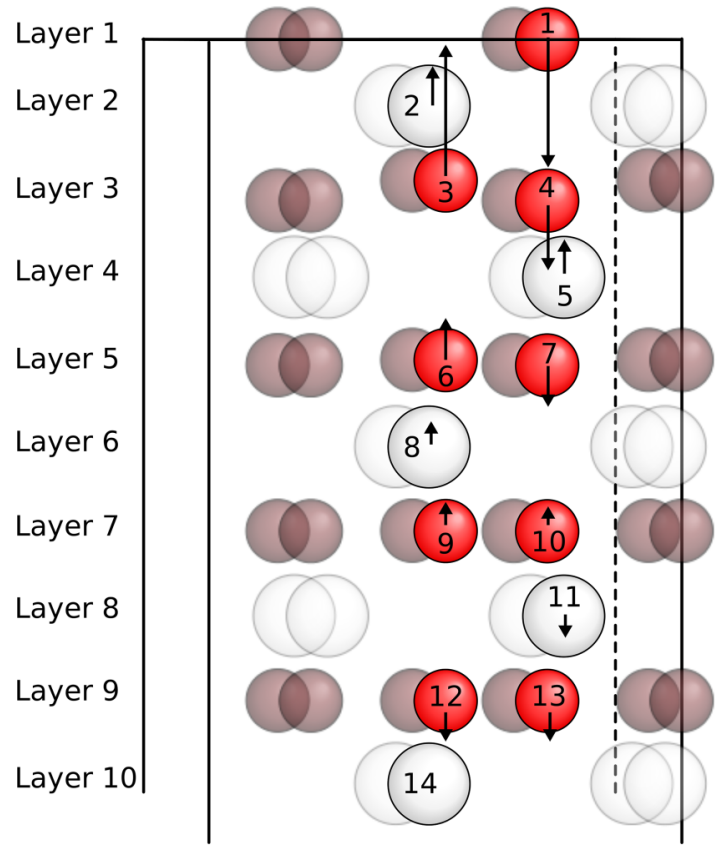

(c)
FIG. 3. (a) Top view onto the (100) $\mathrm{CaF}_{2}$ surface with $\mathrm{F}$ termination (topmost two layers). The cross section area of the unit cell is shaded in dark gray; the cross section area of the simulation box is shaded in light gray (red). (b) Surface top view after removing every second $\mathrm{F}$ ion from the bulk terminated surface. (c) 10 outermost layers of the F-terminated (100) supercell (relaxed) consisting of 19 layers.

\section{SURFACE RECONSTRUCTIONS}

\section{A. (100) $\mathrm{CaF}_{2}$ reconstructions}

We performed structural searches using the MHM, starting from the F-terminated (100) surface since it is lower in energy than the Ca-terminated one (see Table II). This initial structure, shown in Fig. 3(c), is in fact not a local minimum according to our calculations, but a saddle point, which we from here on refer to as SP. Small random displacements of the top layer anions followed by a geometry relaxation leads to a local minimum on the PES and thus a reconstruction of the surface. We performed several MHM simulations to explore further reconstructions of the (100) surface. To reduce the computational cost, we used a thin slab consisting of only seven layers to pre-screen the energy landscape. The five bottom layers were fixed during the whole simulation to imitate the rigid bulk structure. To obtain refined surface energies, the reconstructed atoms from the two top layers found during the structural 


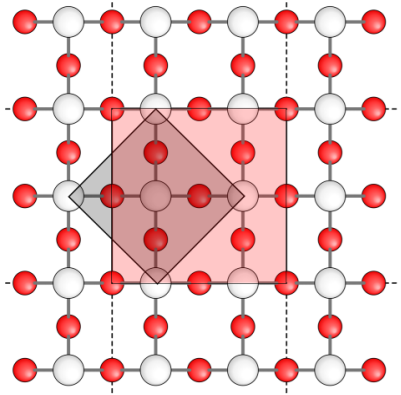

(a)

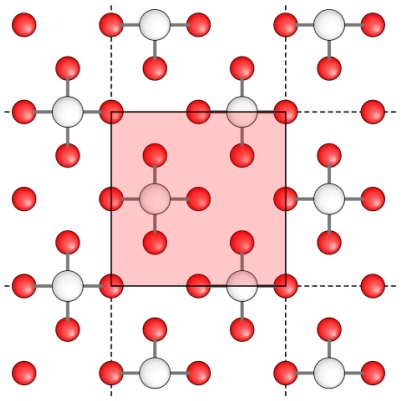

(b)

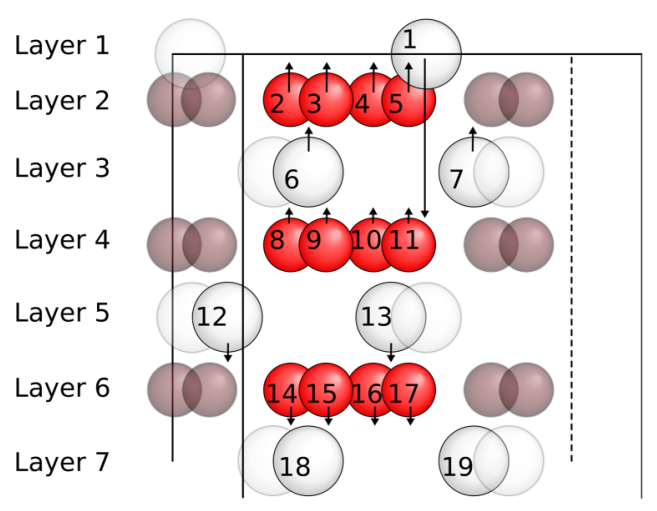

(c)

FIG. 4. (a) Ca-terminated (100) surface (topmost two layers) for $\mathrm{CaF}_{2}$. The cross section area of the unit cell is shaded in dark gray and the supercell area in light gray (red). (b) Surface top view after removing every second $\mathrm{Ca}$ atom of the outermost layers. (c) Seven outermost layers of the relaxed (100) supercell with Ca termination consisting of 13 layers.

search were placed onto the top and the bottom of a larger (100) slab consisting of 11 layers. These final 15 layer slabs were then relaxed, allowing all atoms to move. A similar approach was also employed for the MHM runs of Secs. III C and IV.

The MHM simulations revealed a plethora of reconstructions for the (100) surface, which we categorized into groups

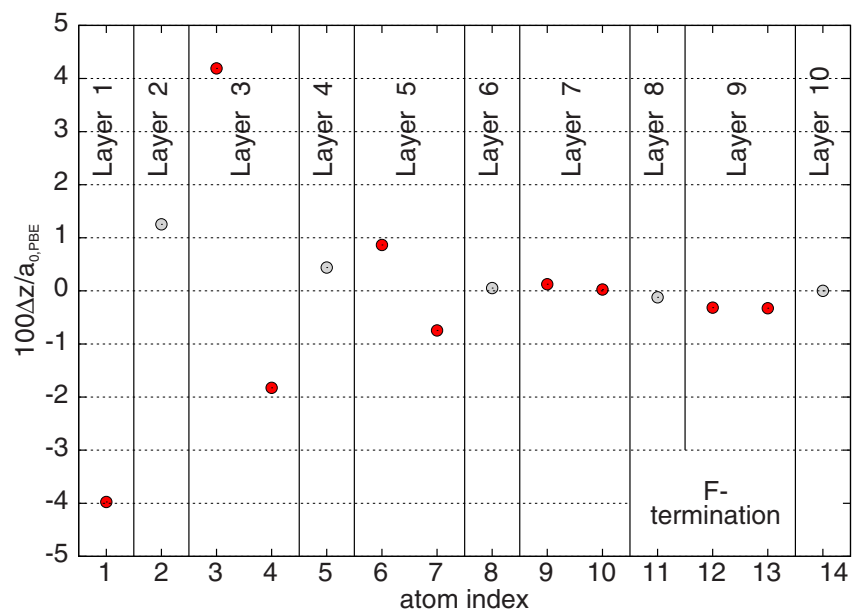

(a) labeled from A to K. Figure 6 summarizes our results (structural data are given in the Supplemental Material [69] ). Members of the same group carry an index X (e.g., AX), which denotes energetically quasidegenerate structures. The labeling of the groups reflects the energetic ordering with respect to LDA results, such that the most stable reconstructions are in group A, the next higher in energy in B, and so on. This ordering slightly differs from LDA to PBE, and the surface energies and reconstruction types are listed in Table III. The energy differences between structures within the same group are very small. The same holds for most barriers connecting different minima of the same group: For example, the height of the barrier connecting B1 and B3 is merely $0.47 \mathrm{~mJ} / \mathrm{m}^{2}$ and only $0.36 \mathrm{~mJ} / \mathrm{m}^{2}$ for the reverse direction. These low barriers between degenerate minima and even between different groups (see Sec. III B) indicate a high mobility of the surface F ions.

All reconstructed surfaces are $\mathrm{F}$ terminated with the exception of J1. Structures A1 and A2 slightly differ in the topmost F-ions position. The central F ions of structure A2 exhibit a buckling relative to the "neighboring" $\mathrm{Ca}$ ions that is not present in structure A1. Based on LDA energies, B members have surface energies that are $1.4 \%$ higher than in group A. The same B members are $3.2 \%$ lower in energy with respect to the A type according to the PBE calculations. Group B reconstructions are variations of the SP structure. The structural difference within the group B members consists in different buckling of the surface anions. B1 is a $c(2 \times 2)$ reconstruction, while the reconstructions in $\mathrm{B} 2$ and $\mathrm{B} 3$ extend over half of the simulation cell. In B2, only every second toplayer anion exhibits a displacement from its SP position, while in B3 the toplayer anions exhibit a pairwise opposite buckling. In general, the magnitude of buckling is weaker for B-type reconstructions optimized with the PBE functional.

The $\mathrm{C} 1$ superstructure turns out to be the most stable configuration with PBE. Figure 7 shows its structure with five $\mathrm{F}$ ions belonging to the outermost layer which are twofold and threefold coordinated to $\mathrm{Ca}$ atoms. $\mathrm{Ca}$ and $\mathrm{F}$ ions belong to the same plane. This is the only reconstruction with five fluorine anions on the topmost layer. As a consequence, the third layer contains seven fluorine atoms, instead of eight

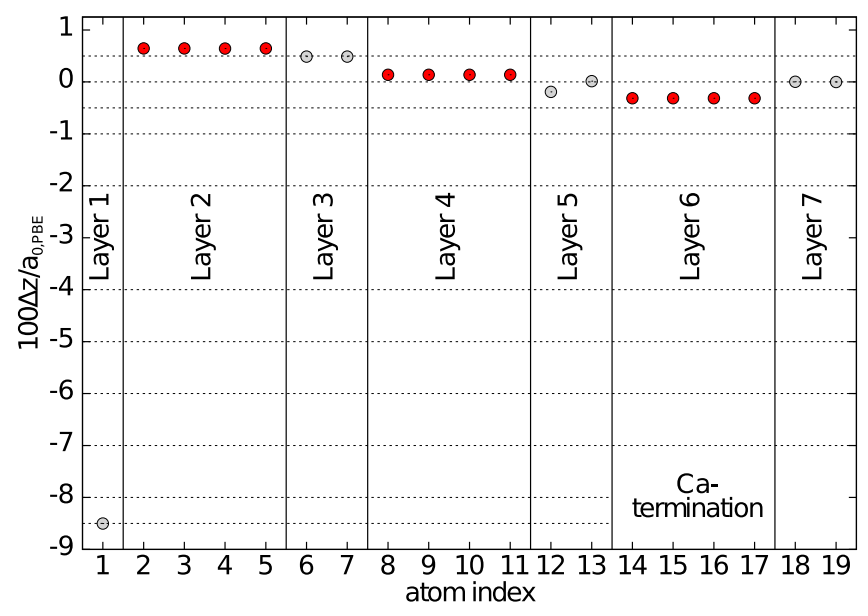

(b)

FIG. 5. Inward/outward relaxation (PBE) amplitudes of the (100) $\mathrm{CaF}_{2}$ slab in units of $\% a_{0, \mathrm{PBE}}$. (a) F-terminated slab consisting of 19 layers; (b) Ca-terminated slab consisting of 13 layers. Atom indexes correspond to Figs. 3(c) and 4(c), respectively. 

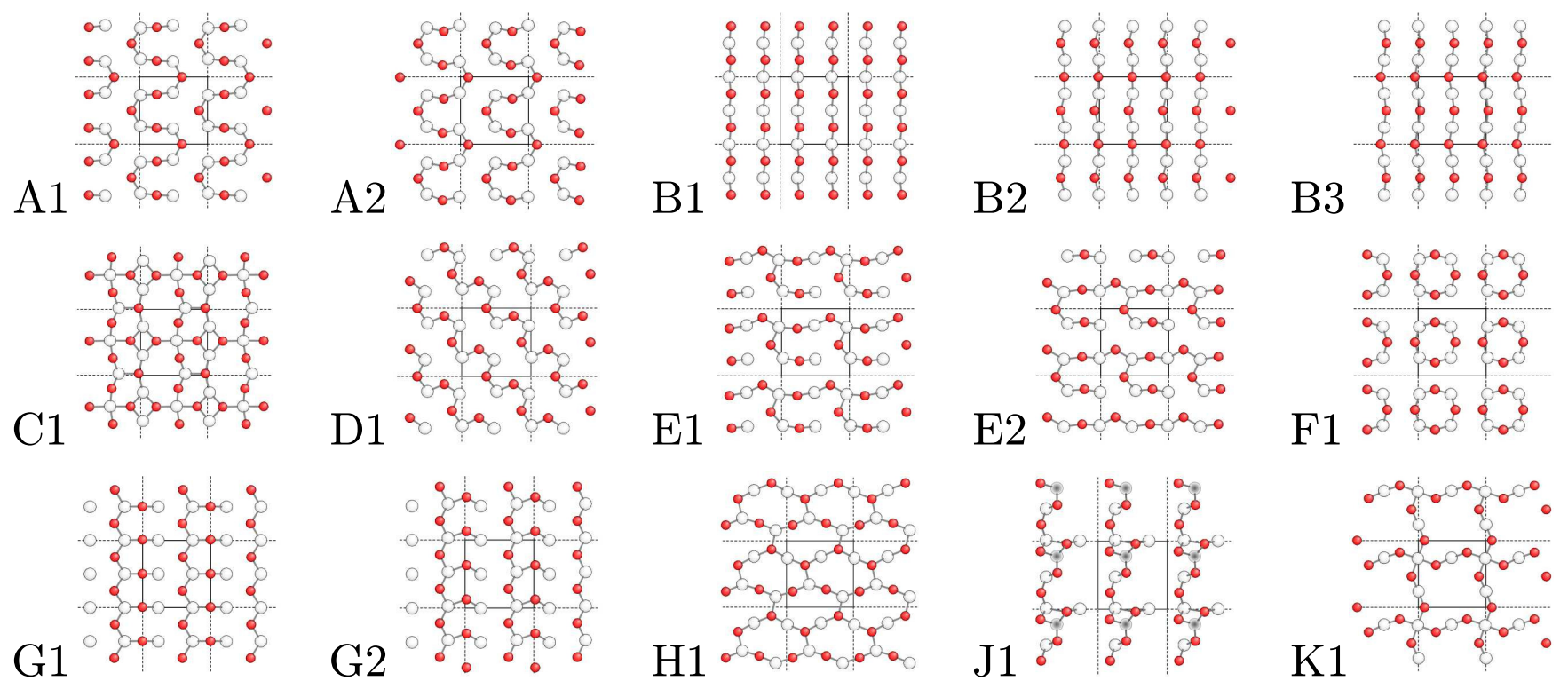

FIG. 6. Normal view onto the (100) reconstructed surfaces of $\mathrm{CaF}_{2}$ (topmost two layers), ordered into groups of energetically degenerate structures. With the exception of structure J1, all surfaces are F terminated. F and Ca atoms are denoted by small dark (red) and large light (gray) spheres. The topmost $\mathrm{Ca}$ atoms in $\mathrm{J} 1$ are denoted by shaded dark-gray spheres.

in all other reconstructions. The surface energy of D1 is about 3.6\% higher than for group A (LDA), and the outmost atoms form a chainlike motif of alternating calcium and fluorine atoms. Group E consists of two superstructures with surface energies $4.8 \%$ higher compared to group A (LDA). Group F consists of a single reconstruction F1. The group $\mathrm{G}$ reconstructions G1 and G2 differ in their buckling of the toplayer anions. The $\mathrm{H} 1$ reconstruction shows a mixed twofold

TABLE III. $\gamma_{(100)}$ surface energies $\left[\mathrm{J} / \mathrm{m}^{2}\right]$ for all (100) $\mathrm{CaF}_{2}$ reconstructions (15 layers slabs) by LDA and PBE calculations. Coordination of ions belonging to the two topmost $\mathrm{F}$ and Ca layers is reported in the fourth and fifth columns: Numbers of the column shows the coordination number of each ion with its next neighbor, while the superscript represents the number of atoms in the layer with such coordination.

\begin{tabular}{lccccc}
\hline \hline structure & $\gamma_{(100, \mathrm{LDA})}$ & $\gamma_{(100, \mathrm{PBE})}$ & $\mathrm{F}$ & $\mathrm{Ca}$ & type \\
\hline $\mathrm{A} 1$ & 1.137 & 0.807 & $2^{4}$ & $6^{4}$ & $(2 \sqrt{2} \times 2 \sqrt{2}) R_{45^{\circ}}$ \\
$\mathrm{A} 2$ & 1.137 & 0.808 & $2^{4}$ & $6^{4}$ & $(2 \sqrt{2} \times 2 \sqrt{2}) R_{45^{\circ}}$ \\
$\mathrm{B} 1$ & 1.153 & 0.782 & $2^{4}$ & $6^{4}$ & $c(2 \times 2)$ \\
$\mathrm{B} 2$ & 1.153 & 0.782 & $2^{4}$ & $6^{4}$ & $(\sqrt{2} \times 2 \sqrt{2}) R_{45^{\circ}}$ \\
$\mathrm{B} 3$ & 1.153 & 0.782 & $2^{4}$ & $6^{4}$ & $(\sqrt{2} \times 2 \sqrt{2}) R_{45^{\circ}}$ \\
$\mathrm{SP}$ & 1.153 & 0.781 & $2^{4}$ & $6^{4}$ & $c(2 \times 2)$ \\
$\mathrm{C} 1$ & 1.160 & 0.729 & $2^{2}, 3^{3}$ & $5^{1} 6^{3}$ & $(2 \sqrt{2} \times 2 \sqrt{2}) R_{45^{\circ}}$ \\
$\mathrm{D} 1$ & 1.178 & 0.883 & $2^{4}$ & $6^{4}$ & $(2 \sqrt{2} \times 2 \sqrt{2}) R_{45^{\circ}}$ \\
$\mathrm{E} 1$ & 1.192 & 0.847 & $2^{4}$ & $5^{1} 6^{3}$ & $(2 \sqrt{2} \times 2 \sqrt{2}) R_{45^{\circ}}$ \\
$\mathrm{E} 2$ & 1.192 & 0.847 & $2^{4}$ & $5^{1} 6^{3}$ & $(2 \sqrt{2} \times 2 \sqrt{2}) R_{45^{\circ}}$ \\
$\mathrm{F} 1$ & 1.234 & 0.884 & $2^{4}$ & $6^{4}$ & $(2 \sqrt{2} \times 2 \sqrt{2}) R_{45^{\circ}}$ \\
$\mathrm{G} 1$ & 1.246 & 0.905 & $2^{4}$ & $5^{2} 7^{2}$ & $(\sqrt{2} \times 2 \sqrt{2}) R_{45^{\circ}}$ \\
$\mathrm{H} 1$ & 1.248 & 0.871 & $2^{2}, 3^{2}$ & $5^{1} 6^{3}$ & $(2 \sqrt{2} \times 2 \sqrt{2}) R_{45^{\circ}}$ \\
$\mathrm{G} 2$ & 1.249 & 0.908 & $2^{4}$ & $5^{2} 7^{2}$ & $(\sqrt{2} \times 2 \sqrt{2}) R_{45^{\circ}}$ \\
$\mathrm{J} 1$ & 1.688 & 0.845 & $2^{2}, 3^{2}$ & $5^{1} 6^{3}$ & $c(2 \times 2)$ \\
$\mathrm{K} 1$ & 1.945 & 1.501 & $2^{4}$ & $4^{1} 6^{2} 8^{1}$ & $(2 \sqrt{2} \times 2 \sqrt{2}) R_{45^{\circ}}$ \\
\hline \hline
\end{tabular}

and threefold coordination for surface anions. Reconstruction $\mathrm{J} 1$ is $\mathrm{Ca}$ terminated and involves therefore a considerable rearrangement of (former) topmost cations. This structure is not stable with PBE and relaxes to a D1-type structure with a different motif for the surface chains (with twofold and threefold coordination for $\mathrm{F}$ anions). Finally, the K1 structure is energetically the least stable. The high barrier connecting the $\mathrm{K} 1$ configuration to other reconstructions makes its existence rather unlikely (see Sec. III B). Note that the surface energies of all reconstructions from A- to H-type spans a range of merely $0.179 \mathrm{~J} / \mathrm{m}^{2}$ which corresponds to 168 $\mathrm{meV}$ per surface atom. Due to the extensive search for low energy surface structures, we are confident to have found all energetically accessible structures given the constraint on the cell size and the number of mobile surface atoms. Nevertheless, different and larger supercells might possibly reveal further reconstruction geometries.

A deeper insight into the energetic ordering of the (100) reconstructions can be gained by investigating the coordination of the atoms in the two topmost $\mathrm{F}$ and $\mathrm{Ca}$ layers. Each layer is formed by four atoms in our periodic cell (periodic in the

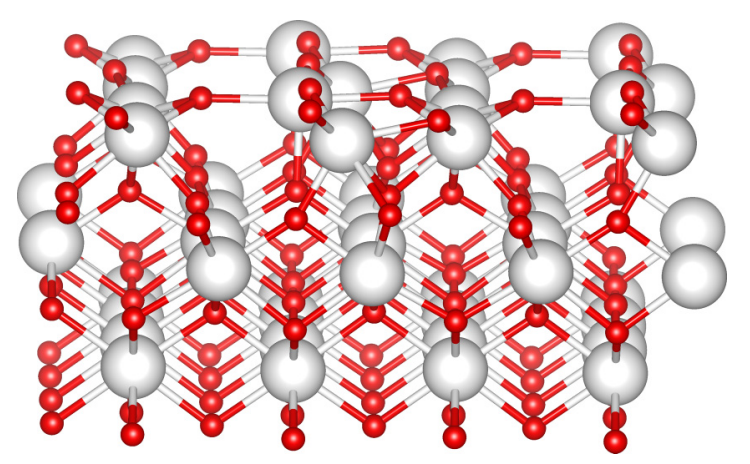

FIG. 7. Lowest energy $\mathrm{C} 1$ reconstruction of the (100) $\mathrm{CaF}_{2}$ surface. 
surface plane). The columns four and five of Table III list the coordination numbers. Their superscripts give the number of atoms in the layer with this coordination. As a consequence, the sum of superscripts for a fixed ion needs to be four, which corresponds to the total number of atoms in each layer. Reconstructions of type A, B, D, F, and SP show a twofold coordination of the $\mathrm{F}$ anions and a sixfold coordination of the $\mathrm{Ca}$ cations. In the bulk, the coordination is 4 and 8 for $\mathrm{F}$ and $\mathrm{Ca}$ atoms, respectively. The most stable structure within $\mathrm{PBE}$, i.e., $\mathrm{C} 1$, has two $\mathrm{F}$ atoms with twofold coordination and three with threefold coordination, while one calcium is fivefold and three are sixfold coordinated. The topmost calcium and fluorine ions lie on the same plane. This structural feature of $\mathrm{C} 1$ closely resembles the (111) surface, where all $\mathrm{F}$ anions are threefold and all $\mathrm{Ca}$ cations are sevenfold coordinated. The $\mathrm{H}$ and $\mathrm{J}$ structures have coordinations similar to $\mathrm{C} 1$ but are higher in energy with the PBE functional. Finally, $\mathrm{K} 1 \mathrm{has}$ twofold coordination of $\mathrm{F}$, while two $\mathrm{Ca}$ ions are sixfold coordinated and the remaining two $\mathrm{Ca}$ are fourfold and eightfold coordinated. The PBE results suggest that a higher coordination of $\mathrm{F}$ anions leads to more stable structures. This conclusion agrees well with the high stability of the (111) surface, which has a lower surface energy and a higher coordination of the topmost surface $\mathrm{F}$ ions.

To compare the (100) surface reconstructions with the (111) surface we also performed MHM simulations on a (111) terminated slab. Only a single high energy reconstruction was found, which is however thermodynamically inaccessible at ambient temperature due to its high surface energy. This finding agrees well with experimental atomic force microscopy (AFM) studies in ultra high vacuum conditions of vacuum-cleaved slabs [70]. Furthermore, the (111) $\mathrm{CaF}_{2}$ termination has been used as a model system to investigate the imaging mechanism of AFM measurements by comparing theory and experiment [71,72]. AFM images reveal a hexagonal arrangement of the topmost $\mathrm{F}$ anions with a regular spacing between them. This suggests that no significant surface reconstruction takes place for the (111) surface termination. In contrast to (111), the large amount of energetically closely spaced surface reconstructions of the (100) face indicates that we are dealing with a highly dynamic system based on a high mobility of the topmost surface anions. Experimentally, there is evidence for such a dynamic environment from low energy electron diffraction (LEED), AFM and transmission electron microscopy (TEM) measurements [5]. A large number of crystallites covering the whole surface have been found for moderate annealing of the (100) termination (annealing promotes ion diffusion, surface discharging, and atomic re-arrangements). No sign of a unique, simple surface motif or superstructure has been so far detected. In overgrowth conditions, microscopic $\{111\}$ pyramids start to appear, indicating that $\mathrm{CaF}_{2}$ prefers to grow through (111) facets in vacuum conditions.

\section{B. Barriers between minima}

The large variety of reconstructions on the (100) surface in a very small energy range suggests that multiple structural arrangements are accessible at finite temperatures. Features of the energy landscape going beyond the energies of the individual local minima are essential to assess the dynamical processes involved during such structural rearrangements. Within the transition state theory, the rate constant $k_{a b}$ from a minimum $a$ to $b$ through a first order transition state $\dagger$ at temperature $T$ is estimated through the energy difference $\Delta V_{a b}$ between the minimum and the transition state by [73]

$$
k_{a b}=\frac{k_{\mathrm{B}} T}{h} \frac{Z^{\dagger}}{Z_{a}} e^{-\Delta V / k_{\mathrm{B}} T},
$$

where $Z_{a}$ and $Z^{\dagger}$ are the partition functions of the local minimum and the transition state, respectively, and $k_{\mathrm{B}}$ is the Boltzmann constant. We approximate the prefactor $\frac{k T}{h} \frac{Z^{\dagger}}{Z_{a}}$ by an attempt frequency $A$ which is of the order of $10^{12} s^{-1}$, such that the only quantities that need to be computed are the barrier heights interconnecting the local minima.

A double ended saddle point search algorithm using the barsaddle method was employed to find transition states between various surface reconstructions [53]. The barrier heights with the LDA exchange-correlation functional are summarized in a disconnectivity graph [73] in Fig. 8 with a threshold value of $40 \mathrm{~mJ} / \mathrm{m}^{2}$. The end of the branches correspond to the energy differences with respect to the A1 reconstruction, and the branch nodes correspond to the lowest barrier heights interconnecting the various reconstructions. Note that four structures, B1, B3, D1, and E1, are connected to the ground state A1 through barriers of less than $120 \mathrm{~mJ} / \mathrm{m}^{2}$ such that one can expect a fluctuation of the surface reconstructions at finite temperature. On the other hand, reconstructions such as F1 or K1 are separated by high barriers from A1 such that they will not be easily accessible in experiments. With the barrier heights at hand it is possible to compute the master equation dynamics [73] by constructing the transition matrix between the various reconstructions from the rate constants. The low barrier heights ensure that the equilibrium distribution is obtained in a time period of the order of $10^{-6} \mathrm{sec}$ at $400 \mathrm{~K}$ for any initial occupation.

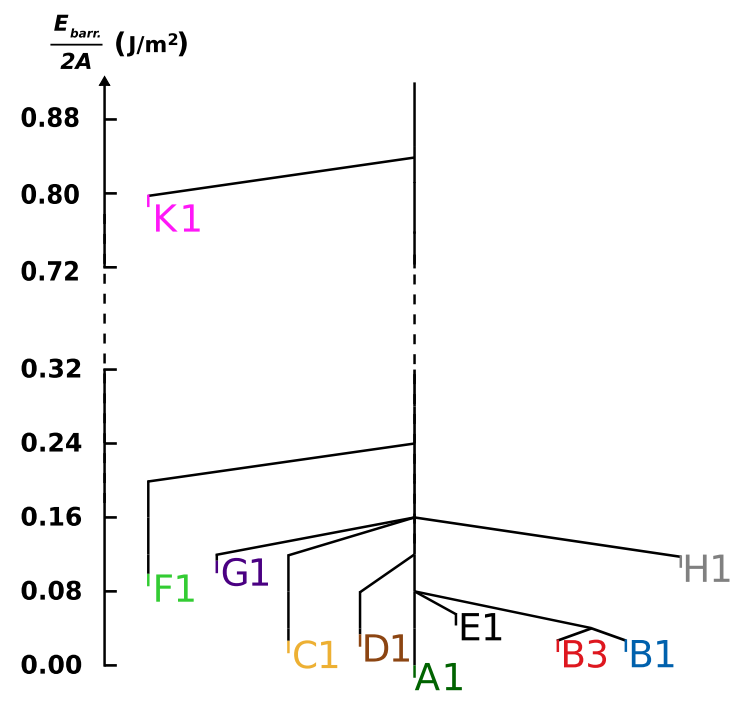

FIG. 8. Disconnectivity graph of the $\mathrm{CaF}_{2}$ (100) reconstructions (LDA calculations). For clarity a threshold value of $40 \mathrm{~mJ} / \mathrm{m}^{2}$ was chosen to bin the saddle point energies. 
TABLE IV. Atomic displacements $\Delta z$ relative to the bulk positions of the atoms in terms of $100 \Delta z / a_{0, \mathrm{PBE}}$ for (100) supercells consisting of 15 layers. Data refer to the fluorites $\mathrm{CaF}_{2}, \mathrm{SrF}_{2}, \mathrm{CdF}_{2}$, and $\mathrm{BaF}_{2}$.

\begin{tabular}{lrrrr}
\hline \hline atom index & $\mathrm{CaF}_{2}$ & $\mathrm{SrF}_{2}$ & $\mathrm{CdF}_{2}$ & $\mathrm{BaF}_{2}$ \\
\hline 1 & -3.1 & -3.7 & -3.9 & -3.9 \\
2 & 1.9 & 1.6 & 1.5 & 2.1 \\
3 & 5.1 & 4.2 & 4.1 & 4.0 \\
4 & -2.2 & -1.3 & -1.8 & 0.0 \\
5 & 0.8 & 0.8 & 0.5 & 1.7 \\
6 & 1.7 & 1.1 & 0.5 & 1.6 \\
7 & -1.1 & -0.3 & 0.0 & 1.4 \\
8 & 0.1 & 0.0 & 0.0 & 1.0 \\
9 & -0.1 & -0.3 & -0.2 & 0.5 \\
10 & -0.4 & -0.4 & -0.3 & 0.5 \\
11 & 0.0 & 0.0 & 0.0 & -0.1 \\
\hline \hline
\end{tabular}

\section{Fluorite crystals $\mathrm{SrF}_{2}, \mathrm{CdF}_{2}$, and $\mathrm{BaF}_{2}$}

Reconstructions of the (100) surface of several other fluorite crystals were also been investigated, namely $\mathrm{SrF}_{2}, \mathrm{CdF}_{2}$, and $\mathrm{BaF}_{2}$. For the cleaved slab shown in Fig. 3 consisting of 15 layers, the atomic displacements $\Delta z$ after a local geometry optimization are comparable to the (100) $\mathrm{CaF}_{2}$ surface and are summarized in terms of $100 \Delta z / a_{0, \mathrm{PBE}}$ in Table IV. The displacements within the top layers are almost identical for all systems under consideration, suggesting that all above compounds, which belong to the same family of ionic materials with the fluorite structure, among other properties, show similar dynamics at the surface. Consequently, common structural motifs are expected to be found between them with similar features of the potential energy surface. To test this hypothesis, we performed additional MHM simulations (PBE functionals) for the (100) surface of $\mathrm{SrF}_{2}, \mathrm{CdF}_{2}$, and $\mathrm{BaF}_{2}$.

Table V summarizes the surface energies $\gamma_{(100)}$ for all reconstructions of the investigated fluorites (coordinate files are

TABLE V. Surface energies $\gamma_{(100)}\left[\mathrm{J} / \mathrm{m}^{2}\right]$ in vacuum for the (100) reconstructions of $\mathrm{SrF}_{2}, \mathrm{CdF}_{2}$, and $\mathrm{BaF}_{2}$ [Eq. (1), $\mathrm{PBE}$ functional].

\begin{tabular}{lccc}
\hline \hline structure & $\mathrm{SrF}_{2}$ & $\mathrm{CdF}_{2}$ & $\mathrm{BaF}_{2}$ \\
\hline $\mathrm{A} 1$ & 0.764 & 0.611 & 0.612 \\
$\mathrm{~A} 2$ & 0.765 & 0.610 & 0.614 \\
$\mathrm{~B} 1$ & 0.764 & 0.546 & 0.616 \\
$\mathrm{~B} 2$ & 0.765 & 0.546 & 0.618 \\
$\mathrm{~B} 3$ & 0.765 & 0.546 & \\
$\mathrm{SP}$ & 0.765 & 0.546 & 0.621 \\
$\mathrm{C} 1$ & 0.768 & 0.561 & 0.652 \\
$\mathrm{D} 1$ & 0.802 & 0.723 & 0.630 \\
$\mathrm{E} 1$ & 0.800 & 0.631 & 0.639 \\
$\mathrm{E} 2$ & 0.800 & 0.619 & 0.639 \\
$\mathrm{~F} 1$ & 0.855 & 0.643 & 0.689 \\
$\mathrm{G} 1$ & 0.836 & 0.692 & 0.664 \\
$\mathrm{G} 2$ & 0.837 & 0.685 & 0.665 \\
$\mathrm{H} 1$ & 0.832 & 0.700 & 0.670 \\
$\mathrm{~J} 1$ & 1.135 & 0.714 & 0.890 \\
$\mathrm{~K} 1$ & 1.419 & & \\
\hline \hline
\end{tabular}

given in the Supplemental Material [69]). As expected, most low-energy reconstructions of $\mathrm{CaF}_{2}$ also exist for the (100) surface of $\mathrm{SrF}_{2}, \mathrm{CdF}_{2}$, and $\mathrm{BaF}_{2}$, with a slightly different energetic ordering. The correspondence between a given reconstruction and the coordination of surface atoms is still maintained. All surfaces are $\mathrm{F}$ terminated and only the group $\mathrm{J}$ structures are cation terminated. Some reconstructions are not present in all fluorites, where their relaxations lead to a different closeby stable minimum. A-, B-, and C-type reconstructions are the most stable for $\mathrm{SrF}_{2}$. All of them are almost degenerate in energy. The global minimum for $\mathrm{CdF}_{2}$ is represented by a B-type structure, namely a variation of the simple cleaved termination. $\mathrm{A} 1$ is the most stable surface for $\mathrm{BaF}_{2}$, although the B-type reconstructions are very close in energy. Group B members of $\mathrm{BaF}_{2}$ and $\mathrm{SrF}_{2}$ exhibit a strong buckling of the top layer $\mathrm{F}$ anions, while the same $\mathrm{B}$ family shows a weak buckling for $\mathrm{CdF}_{2}$. The $\mathrm{K} 1$ reconstruction is stable for $\mathrm{SrF}_{2}$ but not for $\mathrm{CdF}_{2}$ and $\mathrm{BaF}_{2}$. All reconstructions from $\mathrm{A}-\mathrm{H}$ type lie within an energy range of $0.091,0.177$, and $0.077 \mathrm{~J} / \mathrm{m}^{2}$ (96, 165, and $92 \mathrm{meV}$ per surface atom) for $\mathrm{SrF}_{2}, \mathrm{CdF}_{2}$, and $\mathrm{BaF}_{2}$, respectively. These energy ranges are close to the one found for the calcium-fluoride system. Hence, we conclude that the (100) F-terminated surfaces are extremely dynamic systems for all fluorite-type crystals investigated in this paper. A high ion mobility is expected at the surface with a strongly fluctuating coordination number of the topmost atoms.

\section{HYDRATED SURFACES}

Polar surfaces like the ones of $\mathrm{CaF}_{2}$ are well known to exhibit reactive terminations at high and low $\mathrm{pH}$ values. On these surfaces, hydroxyl radicals or protons can easily bind to $\mathrm{Ca}$ cations or $\mathrm{F}$ anions, respectively. Khatib et al. [28] used a combination of advanced phase-resolved vibrational sum frequency generation (VSFG) spectra and $a b$ initio molecular dynamics simulations to provide a quantitative understanding of the (111) fluorite-water interface at the atomistic level. According to their results, the dissolution of fluoride ions is responsible for the positive surface charges at low $\mathrm{pH}$, rather than the adsorption of protons. At high $\mathrm{pH}$ on the other hand they observed the presence of $\mathrm{Ca}-\mathrm{OH}$ species pointing towards the water. At neutral $\mathrm{pH}$, the VSFG signal vanished, indicating a more random orientation of the interfacial water molecules at a near-neutral surface. Water contact angle $\theta_{\mathrm{C}}$ measurements on calcium fluoride (111) surfaces for several $\mathrm{pH}$ conditions confirm this behavior, where $\theta_{\mathrm{C}}$ is nonzero only for near-neutral $\mathrm{pH}$ values [74].

In our study we focused on neutral $\mathrm{pH}$ condition, exploring the effects of a water environment on the (111) surface and (100) reconstructions discussed in the previous sections. We employed both an explicit scheme and an implicit solvation model to include the solvent at the DFT level. By adding explicit water molecules to our simulations, we investigated how water modifies the surface structure at different degrees of coverage. Different coverages were modeled by adding an increasing number of water molecules. In the second approach, the aqueous environment was modeled at the DFT level by means of a recently developed implicit solvation model $[45,46]$ which replaces the explicit water molecules by a continuum body surrounding the quantum-mechanical system. 

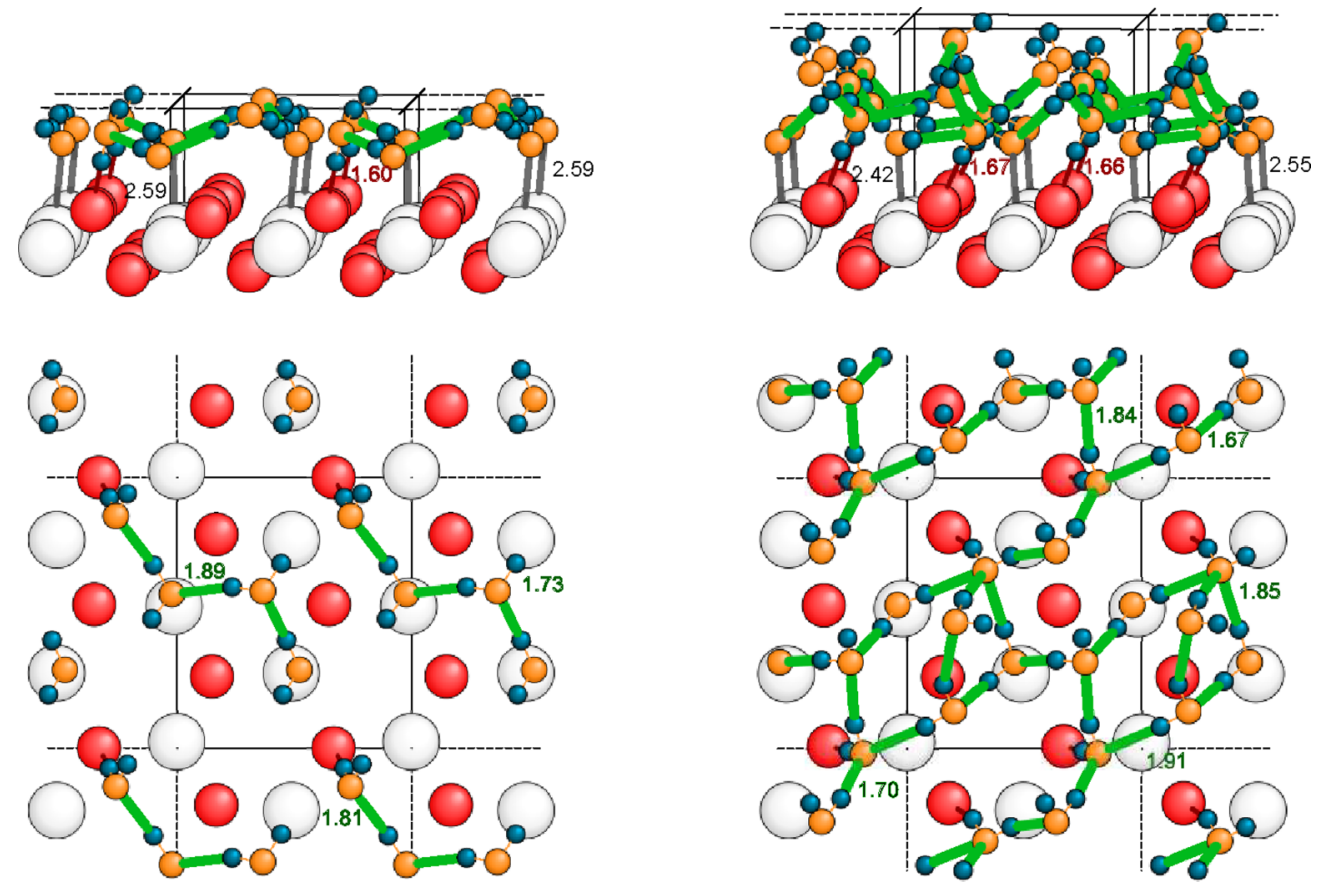

FIG. 9. Structures of the most stable configurations of the hydrated (111) $\mathrm{CaF}_{2}$ surface for $100 \%$ (left) and 200\% coverage (right) in side view (top) and top view (bottom). Top views show only the two topmost anion and cation layers. $\mathrm{H}$ atoms denoted by small dark (blue), $\mathrm{O}$ atoms by medium light gray (yellow), $\mathrm{F}$ atoms by medium dark (red), and Ca atoms by large light-gray (white) spheres. Additionally shown are the $\mathrm{H}-\mathrm{F}$ bonds (thin, red) and the $\mathrm{Ca}-\mathrm{O}$ bonds (bold, dark gray) and hydrogen bonds (bold, green) together with their typical bond lengths in $\AA$.

This implicit approach allowed efficient minima hopping explorations of the potential energy surface for hydrated fluorite terminations. Applying a global search algorithm at the DFT level to a solvated system together with an implicit solvation scheme poses a significant advancement in multiscale modeling.

\section{A. Interface with explicit water molecules}

We investigated the hydrated (111) and (100) $\mathrm{CaF}_{2}$ surfaces with coverages ranging from $25 \%$ to $200 \%$, where a $100 \%$ coverage is defined as one water molecule per $\mathrm{Ca}$ surface cation. Starting from a clean F-terminated surface, we added one water molecule after another to incrementally increase the surface coverage. Since these water molecules can orient in different ways with respect to the surface, different low energy configurations were sampled for each coverage degree by performing MHM simulations. Similar to our studies on the clean surfaces, relatively thin surface slabs were used to prescreen the energy landscape. The final hydrated surface geometries were refined by placing the outmost layers on the top and bottom of a thicker slab of 15 and 11 single layers for (111) and (100) surfaces, respectively (final structures with 20 $\mathrm{CaF}_{2}$ units in both cases).

Figure 9 shows the most stable configurations of the (111) $-\mathrm{CaF}_{2}$-water interface for $100 \%$ and $200 \%$ coverage. Water molecules adsorb almost in a nearly planar way onto the surface and a gap is present between the first water layer and the fluorite surface. The presence of water leads, in contrast to the clean (111) surface, to a surface relaxation by a pairwise opposite buckling of the top-layer anions and cations without further surface reconstructions. Ion-dipole interactions between the topmost $\mathrm{CaF}_{2}$ layer and molecules of the first water layer lead to $\mathrm{Ca}-\mathrm{O}$ and $\mathrm{H}-\mathrm{F}$ bonding. The average $\mathrm{Ca}-\mathrm{O}$ bond length is $2.48 \AA$, while the average $\mathrm{H}-\mathrm{F}$ bond length is $1.64 \AA$. Additionally, several hydrogen bonds are formed among the water molecules with an average bond length of $1.79 \AA$. The low degree of interaction for the (111) termination with water agrees well with previous experimental results through frequency modulation atomic force microscopy (FMAFM) [27] and 3D-AFM [29] measurements. Both techniques are able to resolve the solid-liquid interface on an atomic scale. Microscopy images revealed that the $\mathrm{CaF}_{2}$ (111) surface does not change its structure once in contact with neutral $\mathrm{pH}$ water, exhibiting an atomically clean surface without any adsorbates. Similar conclusions can be deducted from the VSFG spectra [28].

For the (100) termination and a coverage of $25 \%$ (just one water molecule), the MHM predicts a putative global minimum to be an E-type reconstruction with the water oxygen binding as a bridge between two calcium cations (increasing the coordination of two calcium cations, one fivefold and the other sixfold coordinated), closing the surface ring of $\mathrm{Ca}-\mathrm{F}$ ions (see E-type structures in Fig. 6). In general, water molecules in proximity of the fluorite surface mainly interact with the calcium cation through their oxygen atoms. Divalent oxygens of the interfacial water molecules bind to divalent calcium ions. Such an interaction is much stronger than the one of the monovalent fluorine anions with monovalent hydrogens. This trend holds for all water coverages. For 50\% and higher coverages, the MHM predicts that the water molecules adsorb on the group $\mathrm{D}$ (100) reconstructed surface, and the most stable 

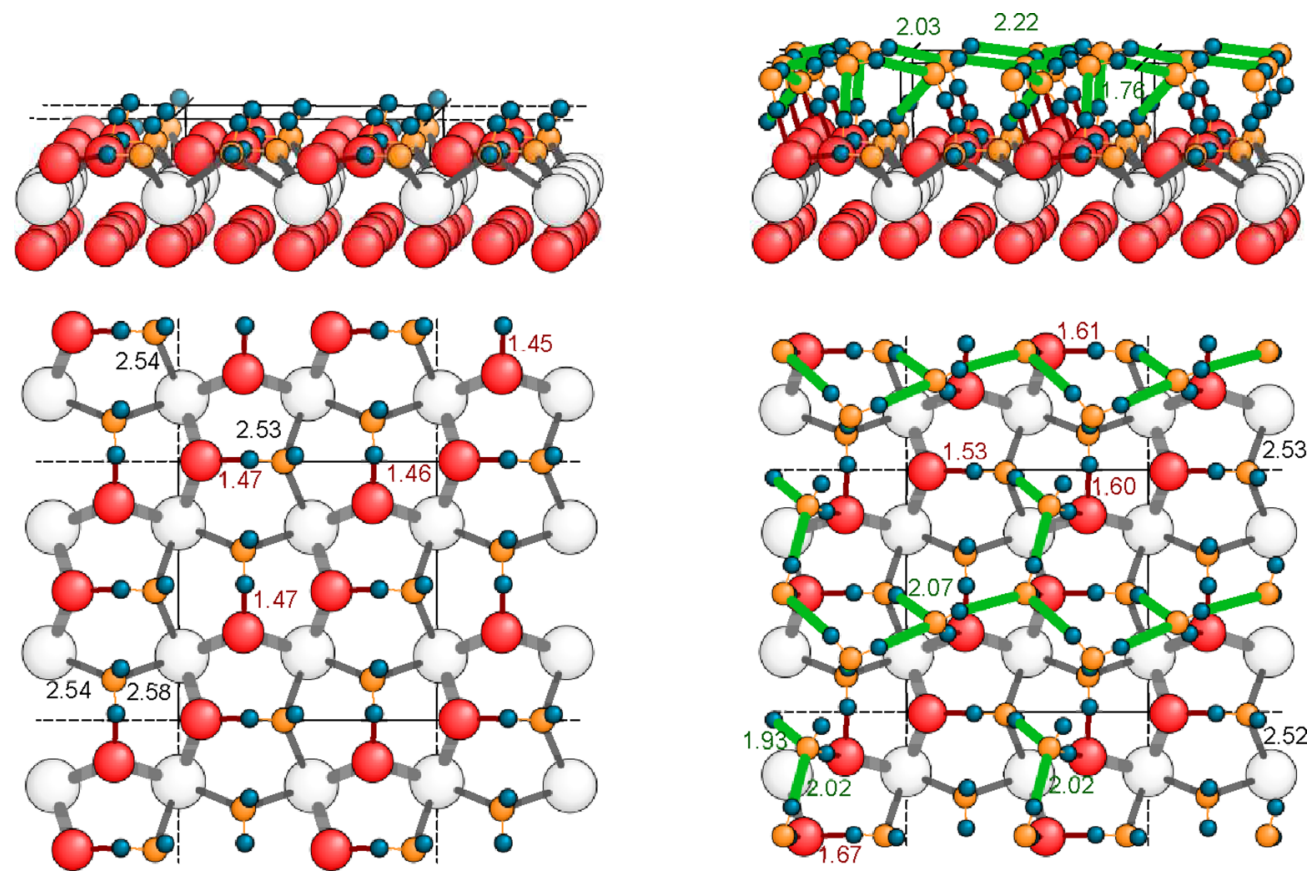

FIG. 10. Structures of the most stable configurations of the hydrated (100) $\mathrm{CaF}_{2}$ surface for $100 \%$ (left) and 200\% coverage (right) in side view (top) and top view (bottom). Color description as Fig. 9. Top views show the underlying reconstructions (bold, dark gray) which are of type D for $100 \%$ and $200 \%$ coverage.

configuration for the (100)- $\mathrm{CaF}_{2}$-water interface is shown in Fig. 10. A perfect D-type reconstruction appears where the oxygen atoms increase the coordination of $\mathrm{Ca}$, forming a bridge between the two nearest cations, while the hydrogen atoms point towards the $\mathrm{F}$ anions.

The reconstructions and the high ion mobility lead to an enhanced hydrophilicity of the (100) surface [30] compared to (111) and allow the water molecules to adsorb in an optimal orientation and location. Water molecules thus become closely packed at the (100) surface. Each water molecule forms a short $\mathrm{H}-\mathrm{F}$ bond and two $\mathrm{Ca}-\mathrm{O}$ bonds with an average bond length of $1.55 \AA$ and $2.55 \AA$, respectively. The number of $\mathrm{Ca}-\mathrm{O}$ bonds is about four times larger than the number of $\mathrm{Ca}-\mathrm{O}$ bonds at the (111)-water interface, indicating a stronger interaction between the adsorbent and adsorbate. The different coordinations of surface ions play the dominant role for water adsorption. $\mathrm{F}$ and $\mathrm{Ca}$ atoms are three and sevenfold coordinate on the (111) surface, respectively, while they are two and sixfold coordinate on (100). Hence, on the (100) surface there is more room for molecular bonding, especially for the strong $\mathrm{Ca}-\mathrm{O}$ bonds.

Note that in all our MHM runs there was no evidence of any dissociation process for the water molecules or surface dissolution with calcium or fluorine ions moving into the solution. Similar conclusions were found in previous molecular dynamics simulations based on density functional theory, suggesting the preference for an associative rather than dissociative adsorption of water molecules [3,75].

Our results agree with $\mathrm{MD}$ simulations of $\mathrm{CaF}_{2}$ interfaces in the presence of water, where a broad distribution of the dipole moment orientation (with respect to the surface normal) has been found for the (111) termination in contrast to a well defined value for (100). By incorporating the results from MD simulations of interfacial water with experimental sum frequency vibrational spectroscopy (SFVS) measurements, Zhang et al. [30] found an exclusion zone at the (111) surface by the presence of a small $3700 \mathrm{~cm}^{-1}$ peak in the SFVS spectra (related to nonhydrogen bonded $\mathrm{OH}$ groups close to the surface). On the other hand, the SFVS spectra of the hydrated (100) surface showed no evidence of the $3700 \mathrm{~cm}^{-1}$ peak, indicating that interfacial water molecules are more ordered at the (100) surface rather than at the (111) surface of fluorite [30]. In contrast to a pure MD run, the MHM is able to efficiently overcome energy barriers. The close agreement of our results with the MD study of Zhang et al. can be explained by the presence of low barriers, which can be overcome even with MD on a time scale that is computationally affordable.

\section{Adsorption energies}

Taking the global minimum for each degree of coverage, we computed the adsorption energy $E_{(h k l) \text {,add. }}$ as the energy difference between the hydrated, fully relaxed slab and the energy of this slab after introducing a vacuum region between water molecules and the surface. The values $E_{(h k l) \text {,add. for }}$ each additionally adsorbed water molecule with increasing coverage are given in Table VI.

We found a mean adsorption energy of $52 \mathrm{~kJ} / \mathrm{mol}$ for the (111) termination, which suggests that water molecules are physisorbed rather than chemisorbed onto the surface. Our results agree with previous $a b$ initio calculations at the same level of theory [3] and with water contact angle measurements. The latter suggest a partial hydrophobic character of (111) fluorite terminations [30,74]. The average adsorption energy at the (111)-water interface is very close to the condensation energy of water, which is between $-45 \mathrm{~kJ} / \mathrm{mol}$ and $-35 \mathrm{~kJ} / \mathrm{mol}$ for temperatures ranging from $0{ }^{\circ} \mathrm{C}$ to $200{ }^{\circ} \mathrm{C}$. For the (100) surface, the larger adsorption energies clearly indicate that 
TABLE VI. Adsorption energies $E_{(h k l) \text { add. }}[\mathrm{kJ} / \mathrm{mol}]$ for an additionally adsorbed water molecule for the hydrated (111) and (100) $\mathrm{CaF}_{2}$ surfaces.

\begin{tabular}{lcc}
\hline \hline coverage & $E_{(111), \text { add. }}$ & $E_{(100) \text {,add. }}$ \\
\hline $0 \%$ & & \\
$25 \%$ & -50 & -109 \\
$50 \%$ & -59 & -90 \\
$75 \%$ & -41 & -93 \\
$100 \%$ & -69 & -88 \\
$125 \%$ & -54 & -51 \\
$150 \%$ & -32 & -55 \\
$175 \%$ & -62 & -67 \\
$200 \%$ & -50 & -91 \\
\hline \hline
\end{tabular}

each water molecule in the first layer strongly interacts with the surface. Because of the penetration of the first water layer into the surface (see Fig. 10), a 100\% coverage of the (100) surface does not fully saturate all adsorption sites at the interface. A second water layer is therefore required to reliably model the surface in an aqueous environment. Adsorption energies for molecules of this additional layer start to converge to the (111) results. The stronger interaction found for the (100) surface agrees with experimental contact angle measurements, where a total spreading of water, corresponding to an hydrophilic character, has been found experimentally [30].

There are several issues to consider when interpreting the results presented here for the explicit water model. These calculations primarily serve to map out a general trend for the stabilization mechanisms and gain insight into the solidliquid interface at the atomistic level. The MHM allows a local exploration of possible water-surface configurations, a necessary task to evaluate adsorption energies of single water molecules on top of a surface. This structural exploration needs to be repeated for every number of water molecules. As we ramp up the coverage rate and the number of molecules included in our simulations, the search for the global minimum structure becomes computationally increasingly challenging. To properly emulate an aqueous environment, the inclusion of at least three to four water layers is required to account for several solvation shells. Even for strongly charged interfaces, water molecules lose their ordered layered structure after roughly 2-3 solvation layers [28,75]. With this large amount of water molecules in our simulation cell, most of the local minima are conformers with slightly different packing of the solvent. Hence, a large portion of the computational effort is spent solely to rearrange the water molecules instead of actually sampling the solid-water interface. The explored local minima are therefore very close in energy arising from different rearrangements of water molecules, and only a small subset consists of configurations where the fluorite-water interface is really different. To address these issues, we used a second method to study the solid-liquid interface by coupling the MHM with an implicit solvent model [76-79], which effectively reduces the large degree of freedom encountered when dealing with an explicit solvent. The implicit solvent model accounts for the entropy contribution to the total free energy through the nonelectrostatic terms. These terms are absent in simulations of wet systems with explicit solvent molecules.

\section{B. Interface with implicit water}

We employed the soft-sphere continuum solvation model $[45,46]$ to explore the solvent-surface interaction by including the solvent as a continuum at the DFT level. Within this approach, the interface between the quantum-mechanical solute and surrounding environment is described by a fully continuum and differentiable permittivity $\epsilon(\mathbf{r})$ function of the atomic coordinates. The cavity is built up with atomic-centered "soft" spheres, with atomic radii proportional by a factor $f$ to their respective van der Waals radii $r^{v d W}$. Nonelectrostatic contributions to the solvation free energy (which represent the nonelectrostatic interactions between the solute and the solvent) linearly depend on the cavity quantum surface by means of a proportional prefactor $\alpha+\gamma$ ( $\gamma$ is the solvent surface tension and $\alpha$ a tunable parameter). Both $\alpha$ and $f$ are fitted to an experimental dataset of solvation free energies of small molecules or to wetting properties of solid-liquid interfaces [46].

Water detains a dielectric constant of 78.36 (experimental value at low frequency and ambient conditions). The soft-sphere model can successfully describe water in several test cases, providing a mean absolute error (MAE) of $1.12 \mathrm{kcal} / \mathrm{mol}$ over a set of experimental solvation free energies of small organic molecules (soft-sphere parameter set for water: $\epsilon_{0}=78.36 ; r_{\mathrm{F}}^{v d W}=1.68 \AA[80], r_{\mathrm{Ca}}^{v d W}=2.23 \AA$ [81]; $\left.\gamma=0.072 \mathrm{~J} / \mathrm{m}^{2} ; f=1.16 ; \alpha+\gamma=11.5 \mathrm{dyn} / \mathrm{cm}\right)$.

We performed MHM runs as described in Sec. III, but now the topmost surface layer was allowed to interact with the implicit water. Minima found in vacuum remain minima on the hydrated surface, indicating that the reconstructions of Fig. 6 still emerge in a water environment. Results of the hydrated (100) reconstructions of $\mathrm{CaF}_{2}$ are reported in Table VII. The spreading coefficient $S$ is also shown, which represents the work performed to spread a liquid over a unit surface area

TABLE VII. Surface energies $\gamma_{(100)}\left[\mathrm{J} / \mathrm{m}^{2}\right]$ in vacuum and in contact with implicit water for the (100) reconstructions of $\mathrm{CaF}_{2}$ [Eq. (1), PBE functional]. The spreading coefficient $S\left[\mathrm{~J} / \mathrm{m}^{2}\right]$ is also reported.

\begin{tabular}{lccc}
\hline \hline & & \multicolumn{2}{c}{$\mathrm{CaF}_{2}$} \\
\cline { 2 - 4 } structure & $\gamma_{(100)}^{\text {vacuum }}$ & $\gamma_{(100)}^{\text {water }}$ & $S$ \\
\hline $\mathrm{A} 1$ & 0.807 & 0.614 & 0.121 \\
$\mathrm{~A} 2$ & 0.808 & 0.614 & 0.121 \\
$\mathrm{~B} 1$ & 0.782 & 0.628 & 0.081 \\
$\mathrm{~B} 2$ & 0.782 & 0.627 & 0.082 \\
$\mathrm{~B} 3$ & 0.782 & 0.627 & 0.082 \\
$\mathrm{SP}$ & 0.781 & 0.627 & 0.081 \\
$\mathrm{C} 1$ & 0.729 & 0.638 & 0.018 \\
$\mathrm{D} 1$ & 0.883 & 0.679 & 0.131 \\
$\mathrm{E} 1$ & 0.847 & 0.660 & 0.114 \\
$\mathrm{E} 2$ & 0.847 & 0.660 & 0.114 \\
$\mathrm{~F} 1$ & 0.884 & 0.615 & 0.196 \\
$\mathrm{G} 1$ & 0.905 & 0.686 & 0.146 \\
$\mathrm{G} 2$ & 0.908 & 0.685 & 0.149 \\
$\mathrm{H} 1$ & 0.871 & 0.748 & 0.050 \\
$\mathrm{~J} 1$ & 0.845 & 0.739 & 0.033 \\
$\mathrm{~K} 1$ & 1.501 & 0.798 & 0.630 \\
\hline \hline
\end{tabular}


of a clean solid (or another liquid) at constant temperature and pressure and in equilibrium with its liquid vapor. It is defined as $S=\gamma_{(h k l)}^{\text {vacuum }}-\gamma^{l g}-\gamma_{(h k l)}^{\text {water }}$, where $\gamma_{(h k l)}^{\text {vacuum }}$ and $\gamma_{(h k l)}^{\text {water }}$ are the surface energies in vacuum and in contact with water, respectively, and $\gamma^{l g}$ is the surface tension of water. Coupled with the Young's equation for the contact angle $\theta_{\mathbf{C}}$, we can derive that $S=\gamma^{l g}\left(\cos \theta_{\mathrm{C}}-1\right)$, indicating that a drop of liquid will partially wet the surface only for $S<0 \mathrm{~J} / \mathrm{m}^{2}$ (hydrophobic), while a total wetting occurs for $S>0 \mathrm{~J} / \mathrm{m}^{2}$ (hydrophilic). Surface energies were computed via Eq. (1) using the PBE functional, and the same equation was employed for wet terminations. For the latter, the expression $E_{\text {slab }}^{\text {relaxed }}$ was used for the total energy of the relaxed slab in contact with implicit water.

Similar reconstructions in water and vacuum differ only by a slight stretching of the surface bonds. The topmost layer distances increase by merely $1-2 \%$ at most, a similar relaxation that also takes place with the explicit water model. A surface energy of 0.365 and $0.299 \mathrm{~J} / \mathrm{m}^{2}$ was found for the (111) surface of $\mathrm{CaF}_{2}$ in vacuum and in contact with water, respectively, clearly indicating that the interaction with the solvent stabilizes the wet interface. A much larger stabilizing effect was found for all (100) reconstructions as reported in Table VII. The decrease in the surface energy of the C1 reconstruction (vacuum global minimum) is $91 \mathrm{~mJ} / \mathrm{m}^{2}$, while for the reconstructions $\mathrm{A} 1$ and $\mathrm{F} 1$ it is even larger with values of 193 and $269 \mathrm{~mJ} / \mathrm{m}^{2}$, respectively. As a consequence, the latter two structures are the lowest energy reconstructions in water. The B-type reconstructions are the next higher in energy by $13 \mathrm{~mJ} / \mathrm{m}^{2}$, while the F1 structure shows the lowest decrease in surface energy during hydration.

The decrease of the surface energy when introducing the water environment correlates with the spreading coefficient $S$. For the (111) surface its value is negative, $S=-6.4 \mathrm{~mJ} / \mathrm{m}^{2}$. In contrast, a positive value of 121 and $18 \mathrm{~mJ} / \mathrm{m}^{2}$ was found for the A-type and C1-type reconstructions. Since $S$ represents the work needed to spread a liquid over a unit surface area, it is clear that the implicit solvation approach correctly reproduces the results in Sec. IV A obtained with the explicit water model. Qualitatively, water interacts more strongly with the (100) terminations than the (111) surface, as indicated by the positive values of $S$ for the former and the negative values for the latter, respectively.

Using the Young's equation, the soft-sphere model predicts a contact angle of $\theta_{\mathrm{C}}=24^{\circ}$, which also agrees well with the experimental reports in the range of $10^{\circ}$ to $42^{\circ}$ for the (111) surface (for different technique) [30,74]. Zhang et al. [30] reported a contact angle of $20^{\circ}$. For the (100) surface, they found $\theta_{\mathrm{C}}=0^{\circ}$, indicating that it is completely hydrophilic. Since our model predicts $S>0 \mathrm{~J} / \mathrm{m}^{2}$ for all reconstructed (100) surfaces, a complete wetting of the hydrated surfaces is expected in agreement with the experimental measurements.

For a (100) Ca-terminated slab (see Fig. 4), the surface energy decreases from $1.211 \mathrm{~J} / \mathrm{m}^{2}$ in vacuum to $0.814 \mathrm{~J} / \mathrm{m}^{2}$ when in contact with water. The MHM with implicit solvent applied to such a Ca-terminated slab does not find any structures lower in energy, suggesting it as global minimum among all Ca-terminated motifs. This strong decrease of $0.397 \mathrm{~J} / \mathrm{m}^{2}$ confirms our explicit water results, showing strong interactions between the surface cations and the oxygens of water. The decrease is in fact larger than the ones for F-terminated reconstructions. The associated spreading coefficient, as a consequence, is $0.324 \mathrm{~J} / \mathrm{m}^{2}$. Although the wetting coefficient is larger than for any F-terminated reconstruction, the A1 reconstruction still retains the lowest interface energy and is therefore the most likely to be observed in experiments.

The two lowest energy hydrated (100) reconstructions, i.e., A1 or F1, are energetically and structurally close to the SP one. A slight shift of the topmost $\mathrm{F}$ anions can readily lead to the F-Ca chain, typical of SP or B-type motifs (see Fig. 6). As we recall, the SP termination represents the straight cleaved bulk termination. Therefore, the aqueous environment renders terminations with low coordination surface atoms more stable. This stabilization of the weak surface reconstruction favors an epitaxial growth of the crystal surface [82]. As a consequence, the growth of the (100) surface is favored at the expense of (111) in wet conditions. Our postulated water-promoted epitaxial growth through (100) terminations agrees with experimental studies of $\mathrm{CaF}_{2}$ grown in near-neutral $\mathrm{pH}$ water which resulted only in cubic structures with (100) surfaces [34]. Several studies had proposed a water-adsorption mechanism to explain the predominant formation of (100) $\mathrm{CaF}_{2}$ faces during crystal growth and dissolution in aqueous environments [31,32]. Glikin [31] suggested an adsorption mechanism where $\mathrm{Ca}^{2+}$ cations and $\mathrm{H}_{2} \mathrm{O}$ molecules are linked by means of hydroxide ions $\mathrm{OH}^{-}$. Due to the higher $\mathrm{Ca}^{2+}$ density, such a mechanism is more efficient on (100) faces than on (111) or (110) terminations. Our results based on the implicit solvent model go one step further and support a picture where even a neutral aqueous solvent alone promotes the formation of (100) faces [32].

Finally, we extended our studies on the (100) solid-liquid interface for the three additional fluorites, i.e., $\mathrm{SrF}_{2}, \mathrm{CdF}_{2}$, and $\mathrm{BaF}_{2}$. For $\mathrm{SrF}_{2}$, the two lowest energy reconstructions in water are still of type A or B, like in vacuum. Similarly, for $\mathrm{CdF}_{2}$, B-type structures are the lowest energy surfaces both in vacuum and water, while for $\mathrm{BaF}_{2}$ the minimum changes from the A1 termination in vacuum to the degenerate B- or F-type structure in water although their energy difference is merely around $10 \mathrm{~mJ} / \mathrm{m}^{2}$. Considering the structural similarity with calcium fluorite, the interface of the additional fluorites with water should follow similar trends. However, the amount of experimental data in the literature on wettability and similar properties is rather limited. Future experiments can take advantage of our analysis both in vacuum and in the presence of an aqueous solvent.

\section{CONCLUSIONS}

In summary, we performed extensive structural searches for the reconstructed (100) surface of the fluorites $\mathrm{CaF}_{2}, \mathrm{SrF}_{2}$, $\mathrm{CdF}_{2}$, and $\mathrm{BaF}_{2}$. A plethora of low-energy reconstructions were discovered, most of which are energetically close to each other and in some cases even degenerate. Our structure prediction for fluorite systems is evidence for strong surface reconstruction for ionic systems. Furthermore, many of these structures are connected by very low barriers, resulting in a high mobility of the surface ions. Due to these low barriers, 
several structural motifs are expected to compete with each other at finite temperature, rendering the reconstructed (100) surface a very dynamic system. Our results give an explanation on an atomistic scale of experimental AFM, LEED, and TEM measurements. In these experiments, a large number of crystallites covering the whole surface were found on the (100) $\mathrm{CaF}_{2}$ surface with no sign of a unique, simple surface motif or superstructure.

The high mobility of the surface ions and their lower coordination lead to a stronger interaction at the interface between $\mathrm{CaF}_{2}$ (100) and water compared to the (111) surface. Structural searches for several degrees of water coverage were used to study the solid-liquid interface at the atomistic level. Our results agree with experimental SFVS spectra in literature, which show a random distribution for the first water layer of hydrated (111) surfaces and a more ordered first solvation shell for the (100) termination. These results also agree with the hydrophobic and hydrophilic character of the (111) and (100) surfaces, respectively. The results of the explicit and implicit models agree well with each other, as well as with experimental measurements of wetting properties, including the water contact angles. Our structure prediction search coupled with the implicit solvation model reveals that the water environment makes reconstructions with low coordination surface atoms more stable, promoting (100) instead of (111) growth. The presented work highlights the power of combining structural search at the DFT level with the soft-sphere implicit solvation model, allowing the exploration of the PES of large systems like a wet surface at an affordable computational cost.

\section{ACKNOWLEDGMENTS}

This work was done within the PASC and NCCR MARVEL projects. Computer resources were provided by the Swiss National Supercomputing Centre (CSCS) under Project ID s707 and s700. L.G. acknowledges support from the EXTMOS EU project. M.A. acknowledges support from the Novartis Universität Basel Excellence Scholarship for Life Sciences and the Swiss National Science Foundation (P300P2-158407, P300P2-174475). We thank the Nano Argovia program and SNF for financial support.
[1] N. Sata, K. Eberman, K. Eberl, and J. Maier, Nature (London) 408, 946 (2000).

[2] H. Shi, R. I. Eglitis, and G. Borstel, Phys. Status Solidi B 242, 2041 (2005).

[3] N. H. de Leeuw and T. G. Cooper, J. Mater. Chem. 13, 93 (2003).

[4] T. Sumiya, T. Miura, H. Fujinuma, and S. ichiro Tanaka, Surf. Sci. 376, 192 (1997).

[5] V. Deuster, M. Schick, T. Kayser, H. Dabringhaus, H. Klapper, and K. Wandelt, J. Cryst. Growth 250, 313 (2003).

[6] R. Kaschner, J. Becker, and G. Seifert, Int. J. Mass Spectrom. 176, 103 (1998).

[7] H. Dabringhaus and K. Wandelt, Surf. Sci. 526, 257 (2003).

[8] K. Kawano, T. Ohya, T. Tsurumi, K. Katoh, and R. Nakata, Phys. Rev. B 60, 11984 (1999).

[9] J. Mouchovski, I. Haltakov, and V. Lyutskanov, J. Cryst. Growth 162, 79 (1996).

[10] Q.-Z. Zhao, J.-R. Qiu, X.-W. Jiang, C.-J. Zhao, and C.-S. Zhu, Opt. Express 12, 742 (2004).

[11] B. Zhu, J. Mater. Sci. Lett. 18, 1039 (1999).

[12] A. W. Vere, K. J. Mackey, D. C. Rodway, P. C. Smith, D. M. Frigo, and D. C. Bradley, Adv. Mater. 1, 399 (1989).

[13] S. Santra and A. Malhotra, Wiley Interdisciplinary Reviews: Nanomedicine and Nanobiotechnology 3, 501 (2011).

[14] M. D. Weir, J. L. Moreau, E. D. Levine, H. E. Strassler, L. C. Chow, and H. H. Xu, Dent. Mater. 28, 642 (2012).

[15] J. L. Moreau, M. D. Weir, A. A. Giuseppetti, L. C. Chow, J. M. Antonucci, and H. H. K. Xu, J. Biomed. Mater. Res. Part B: Applied Biomaterials 100B, 1264 (2012).

[16] J. Koeser, T. Carvalho, U. Pieles, and A. Lussi, J. Mater. Sci.: Mater. Med. 25, 1671 (2014).

[17] M. Anji Reddy and M. Fichtner, J. Mater. Chem. 21, 17059 (2011).

[18] C. Rongeat, M. A. Reddy, R. Witter, and M. Fichtner, J. Phys. Chem. C 117, 4943 (2013).
[19] C. Rongeat, M. Anji Reddy, R. Witter, and M. Fichtner, ACS Appl. Mater. Interfaces 6, 2103 (2014).

[20] N. I. Sorokin and B. P. Sobolev, Crystallogr. Rep. 52, 842 (2007).

[21] C. V. Putnis and E. Ruiz-Agudo, Elements 9, 177 (2013).

[22] A. Putnis, Science 343, 1441 (2014).

[23] V. Saxena and S. Ahmed, Environ. Geol. 40, 1084 (2001).

[24] K. Oura, V. Lifshits, A. Saranin, A. Zotov, and M. Katayama, Surface Science: An Introduction, Advanced Texts in Physics (Springer, Berlin, Heidelberg, 2013).

[25] K. Voïtchovsky, J. J. Kuna, S. A. Contera, E. Tosatti, and F. Stellacci, Nat. Nanotechnol. 5, 401 (2010).

[26] M. Watkins and A. L. Shluger, Phys. Rev. Lett. 105, 196101 (2010).

[27] N. Kobayashi, S. Itakura, H. Asakawa, and T. Fukuma, J. Phys. Chem. C 117, 24388 (2013).

[28] R. Khatib, E. H. G. Backus, M. Bonn, M.-J. Perez-Haro, M.-P. Gaigeot, and M. Sulpizi, Sci. Rep. 6, 24287 (2016).

[29] K. Miyazawa, M. Watkins, A. L. Shluger, and T. Fukuma, Nanotechnology 28, 245701 (2017).

[30] X. Zhang, X. Wang, and J. D. Miller, Surface Innovations 3, 39 (2015).

[31] A. E. Glikin, in Polymineral-Metasomatic Crystallogenesis (Springer Netherlands, Dordrecht, 2009), p. 266.

[32] W. A. Franke, Eur. J. Mineral. 27, 255 (2015).

[33] Z. Yang, G. Wang, Y. Guo, F. Kang, Y. Huang, and D. Bo, Mater. Res. Bull. 47, 3965 (2012).

[34] E. Kiryanova and A. Glikin, J. Cryst. Growth 198-199, Part 1, 697 (1999).

[35] G. Wulff, Zeitschrift für Krystallographie und Mineralogie 34, 449 (1901).

[36] R. I. Eglitis, H. Shi, and G. Borstel, Surf. Rev. Lett. 13, 149 (2006).

[37] H. Shi, R. I. Eglitis, and G. Borstel, Phys. Rev. B 72, 045109 (2005). 
[38] The repeat unit in the nonperiodic direction consists of two alternately charged planes carrying the charges $-Q,+Q$, resulting in a nonvanishing dipole moment. According to Ref. [39] this stacking is referred to as type 3. In contrast, the $-Q,+2 Q,-Q$ triple layer stacking of fluorites along (111) with vanishing net-dipole moment is referred to as type 2 .

[39] P. W. Tasker, J. Phys. C: Solid State Phys. 12, 4977 (1979).

[40] P. Hartman, On the Crystal Habit of Fluorite (Bulg. Acad. Sci., Geol. Inst., Mineral Genesis, Sofia, 1974), pp. 111-116.

[41] C. Noguera, J. Phys.: Condens. Matter 12, R367 (2000).

[42] M. Sprik, J. Hutter, and M. Parrinello, J. Chem. Phys. 105, 1142 (1996).

[43] J. C. Grossman, E. Schwegler, E. W. Draeger, F. Gygi, and G. Galli, J. Chem. Phys. 120, 300 (2004).

[44] J. VandeVondele, F. Mohamed, M. Krack, J. Hutter, M. Sprik, and M. Parrinello, J. Chem. Phys. 122, 014515 (2005).

[45] G. Fisicaro, L. Genovese, O. Andreussi, N. Marzari, and S. Goedecker, J. Chem. Phys. 144, 014103 (2016).

[46] G. Fisicaro, L. Genovese, O. Andreussi, S. Mandal, N. N. Nair, N. Marzari, and S. Goedecker, J. Chem. Theory Comput. 13, 3829 (2017).

[47] S. Goedecker, J. Chem. Phys. 120, 9911 (2004).

[48] M. Amsler and S. Goedecker, J. Chem. Phys. 133, 224104 (2010).

[49] S. Roy, S. Goedecker, and V. Hellmann, Phys. Rev. E 77, 056707 (2008).

[50] M. Amsler, S. Botti, M. A. L. Marques, and S. Goedecker, Phys. Rev. Lett. 111, 136101 (2013).

[51] S. A. Ghasemi, S. Goedecker, A. Baratoff, T. Lenosky, E. Meyer, and H. J. Hug, Phys. Rev. Lett. 100, 236106 (2008).

[52] M. Amsler, S. A. Ghasemi, S. Goedecker, A. Neelov, and L. Genovese, Nanotechnology 20, 445301 (2009).

[53] B. Schaefer, S. Mohr, M. Amsler, and S. Goedecker, J. Chem. Phys. 140, 214102 (2014).

[54] L. Genovese, A. Neelov, S. Goedecker, T. Deutsch, S. A. Ghasemi, A. Willand, D. Caliste, O. Zilberberg, M. Rayson, A. Bergman, and R. Schneider, J. Chem. Phys. 129, 014109 (2008).

[55] L. Genovese, B. Videau, M. Ospici, T. Deutsch, S. Goedecker, and J.-F. Mhaut, Comptes Rendus Mécanique 339, 149 (2011).

[56] http://www.bigdft.org.

[57] C. Hartwigsen, S. Goedecker, and J. Hutter, Phys. Rev. B 58, 3641 (1998).

[58] M. Krack, Theor. Chem. Acc. 114, 145 (2005).

[59] K. Burke, J. P. Perdew, and M. Ernzerhof, J. Chem. Phys. 109, 3760 (1998).

[60] M. A. Marques, M. J. Oliveira, and T. Burnus, Comput. Phys. Commun. 183, 2272 (2012).
[61] R. Wyckoff, Crystal Structures, 9th ed., Vol. 1 (Interscience/ Wiley, New York, 1963).

[62] R. T. Downs and M. Hall-Wallace, Am. Mineral. 88, 247 (2003).

[63] L. Gerward, J. S. Olsen, S. Steenstrup, M. Malinowski, S. Åsbrink, and A. Waskowska, J. Appl. Crystallogr. 25, 578 (1992).

[64] S. Speziale and T. S. Duffy, Phys. Chem. Miner. 29, 465 (2002).

[65] Janaf Thermodynamical Tables, J. Phys. Chem. Ref. Data 14 (Suppl. 1) (1985).

[66] C. R. A. Catlow, J. D. Comins, F. A. Germano, R. T. Harley, and W. Hayes, J. Phys. C 11, 3197 (1978).

[67] H. M. Haendler and W. J. Bernard, J. Am. Chem. Soc. 73, 5218 (1951).

[68] V. E. Puchin, A. V. Puchina, M. Huisinga, and M. Reichling, J. Phys.: Condens. Matter 13, 2081 (2001).

[69] See Supplemental Material at http://link.aps.org/supplemental/ 10.1103/PhysRevMaterials.1.033609 for the structural data of all (100) surface reconstructions for $\mathrm{CaF}_{2}, \mathrm{SrF}_{2}, \mathrm{CdF}_{2}$, and $\mathrm{BaF}_{2}$.

[70] J. Engelhardt, H. Dabringhaus, and K. Wandelt, Surf. Sci. 448, 187 (2000).

[71] A. S. Foster, C. Barth, A. L. Shluger, and M. Reichling, Phys. Rev. Lett. 86, 2373 (2001).

[72] A. S. Foster, C. Barth, A. L. Shluger, R. M. Nieminen, and M. Reichling, Phys. Rev. B 66, 235417 (2002).

[73] D. Wales, Energy Landscapes: Applications to Clusters, Biomolecules and Glasses, 1st ed. (Cambridge University Press, Cambridge, 2004).

[74] J. Zawala, J. Drzymala, and K. Malysa, Int. J. Miner. Process. 88, 72 (2008).

[75] M. Watkins, M. L. Berkowitz, and A. L. Shluger, Phys. Chem. Chem. Phys. 13, 12584 (2011).

[76] J. Tomasi and M. Persico, Chem. Rev. 94, 2027 (1994).

[77] J. Tomasi, B. Mennucci, and R. Cammi, Chem. Rev. 105, 2999 (2005).

[78] C. J. Cramer and D. G. Truhlar, Chem. Rev. 99, 2161 (1999).

[79] J.-L. Fattebert and F. Gygi, J. Comput. Chem. 23, 662 (2002).

[80] A. K. Rappe, C. J. Casewit, K. S. Colwell, W. A. Goddard III, and W. M. Skiff, J. Am. Chem. Soc. 114, 10024 (1992).

[81] For calcium we used a van der Waals radius of $2.23 \AA$ which better represents the $\mathrm{CaF}_{2}$ wetting properties in terms of the contact angle. Van der Waals radii of $2.23,1.88$, and $2.23 \AA$ were used for $\mathrm{Sr}, \mathrm{Cd}$, and $\mathrm{Ba}$, respectively. There is no general consensus about their values in the context of implicit solvation models [80]. Our approach, based on the surface wettability [46], allows us, however, to find reliable parameters.

[82] C. Bunn, Proc. Roy. Soc. A 141, 567 (1933). 